\title{
TOI 122b and TOI 237b: Two Small Warm Planets Orbiting Inactive M dwarfs Found by TESS
}

William C. Waalkes ${ }^{1,31}$ (D), Zachory K. Berta-Thompson ${ }^{1}$ (D), Karen A. Collins ${ }^{2}$ (D), Adina D. Feinstein ${ }^{3,31}$ (D), Benjamin M. Tofflemire $^{4}$ (D), Bárbara Rojas-Ayala ${ }^{5}$ (D), Michele L. Silverstein ${ }^{6,7,32}$ (iD), Elisabeth Newton ${ }^{8}$ (D), George R. Ricker ${ }^{9}$ (ID), Roland Vanderspek $^{9}$ (D) , David W. Latham ${ }^{2}$ (D), S. Seager ${ }^{9,10,11}$ (D) , Joshua N. Winn ${ }^{12}$ (D) , Jon M. Jenkins ${ }^{13}$ (D), Jessie Christiansen ${ }^{14}$ (D), Robert F. Goeke $^{9}$ (D), Alan M. Levine ${ }^{9}$ (D) H. P. Osborn ${ }^{9,15}$ (D), S. A. Rinehart ${ }^{16}$ (D), Mark E. Rose ${ }^{13}$ (D), Eric B. Ting ${ }^{13}$ (iD),

Joseph D. Twicken $^{13,17}$ (D), Khalid Barkaoui ${ }^{18,19}$ (D) , Jacob L. Bean ${ }^{3}$ (D) , César Briceño ${ }^{20}$ (D), David R. Ciardi ${ }^{14}$ (iD),

Kevin I. Collins ${ }^{21}$ (D), Dennis Conti ${ }^{22}$ (D), Tianjun Gan $^{23}$ (D), Michaël Gillon ${ }^{18}$ (D), Giovanni Isopi ${ }^{24}$, Emmanuël Jehin ${ }^{25}$ (D),

Eric L. N. Jensen ${ }^{26}$ (D) John F. Kielkopf ${ }^{27}$ (D), Nicholas Law $^{28}$ (D) , Franco Mallia ${ }^{24}$, Andrew W. Mann ${ }^{28}$ (iD),

Benjamin T. Montet ${ }^{29,33}$ (ID), Francisco J. Pozuelos ${ }^{18,25}$ (D) , Howard Relles ${ }^{2}$, Jessica E. Libby-Roberts ${ }^{1}$ (D), and Carl Ziegler ${ }^{30}$ (ID

${ }^{1}$ Department of Astrophysical \& Planetary Sciences, University of Colorado Boulder, 2000 Colorado Avenue, Boulder, CO 80309, USA

${ }^{2}$ Center for Astrophysics | Harvard \& Smithsonian, 60 Garden Street, Cambridge, MA 02138, USA

${ }^{3}$ Department of Astronomy and Astrophysics, University of Chicago, 5640 S. Ellis Avenue, Chicago, IL 60637, USA

${ }^{4}$ Department of Astronomy, The University of Texas at Austin, Austin, TX 78712, USA

5 Instituto de Alta Investigación, Universidad de Tarapacá, Casilla 7D, Arica, Chile

${ }^{6}$ NASA Goddard Space Flight Center, Greenbelt, MD 20771, USA

${ }^{7}$ RECONS Institute, Chambersburg, PA 17201, USA

${ }^{8}$ Department of Physics and Astronomy, Dartmouth College, Hanover NH 03755, USA

${ }^{9}$ Department of Physics and Kavli Institute for Astrophysics and Space Research, Massachusetts Institute of Technology, Cambridge, MA 02139, USA

${ }^{10}$ Department of Earth, Atmospheric and Planetary Sciences, Massachusetts Institute of Technology, Cambridge, MA 02139, USA

${ }^{11}$ Department of Aeronautics and Astronautics, MIT, 77 Massachusetts Avenue, Cambridge, MA 02139, USA

${ }^{12}$ Department of Astrophysical Sciences, Princeton University, 4 Ivy Lane, Princeton, NJ 08544, USA ${ }^{13}$ NASA Ames Research Center, Moffett Field, CA, 94035, USA

${ }^{14}$ Caltech/IPAC-NASA Exoplanet Science Institute, 770 S. Wilson Avenue, Pasadena, CA 91106, USA

${ }^{15}$ NCCR/PlanetS, Centre for Space \& Habitability, University of Bern, Bern, Switzerland

${ }^{16}$ NASA HQ, Planetary Science Division 202-358-1884, USA

${ }^{17}$ SETI Institute, Mountain View, CA 94043, USA

${ }^{18}$ Astrobiology Research Unit, Université de Liège, 19C Allèe du 6 Août, B-4000 Liège, Belgium

${ }^{19}$ Oukaimeden Observatory, High Energy Physics and Astrophysics Laboratory, Cadi Ayyad University, Marrakech, Morocco

${ }^{20}$ Cerro Tololo Interamerican Observatory/NSF's National Optical-Infrared Astronomy Research Laboratory, Casilla 603, La Serena, Chile

${ }^{21}$ George Mason University, 4400 University Drive, Fairfax, VA 22030, USA

${ }^{22}$ American Association of Variable Star Observers, 49 Bay State Road, Cambridge, MA 02138, USA

${ }^{23}$ Department of Astronomy and Tsinghua Centre for Astrophysics, Tsinghua University, Beijing 100084, People's Republic of China

${ }^{24}$ Campo Catino Astronomical Observatory, Regione Lazio, Guarcino (FR) I-03010, Italy

${ }^{25}$ Space Sciences, Technologies and Astrophysics Research (STAR) Institute, Université de Liège, 19C Allèe du 6 Août, B-4000 Liège, Belgium

${ }^{26}$ Dept. of Physics \& Astronomy, Swarthmore College, Swarthmore, PA 19081, USA

${ }^{27}$ Department of Physics and Astronomy, University of Louisville, Louisville, KY 40292, USA

${ }^{28}$ Department of Physics and Astronomy, The University of North Carolina at Chapel Hill, Chapel Hill, NC 27599-3255, USA

${ }^{29}$ School of Physics, University of New South Wales, Sydney NSW 2052, Australia

${ }^{30}$ Dunlap Institute for Astronomy and Astrophysics, University of Toronto, $50 \mathrm{St}$. George Street, Toronto, Ontario M5S 3H4, Canada

Received 2020 April 23; revised 2020 October 2; accepted 2020 October 20; published 2020 December 7

\begin{abstract}
We report the discovery and validation of TOI 122b and TOI 237b, two warm planets transiting inactive M dwarfs observed by the Transiting Exoplanet Survey Satellite (TESS). Our analysis shows that TOI $122 \mathrm{~b}$ has a radius of $2.72 \pm 0.18 R_{\oplus}$ and receives $8.8 \pm 1.0$ times Earth's bolometric insolation, and TOI 237b has a radius of $1.44 \pm 0.12 R_{\oplus}$ and receives $3.7 \pm 0.5$ times Earth's insolation, straddling the $6.7 \times$ Earth insolation that Mercury receives from the Sun. This makes these two of the cooler planets yet discovered by TESS, even on their 5.08 and 5.43 day orbits. Together, they span the small-planet radius valley, providing useful laboratories for exploring volatile evolution around $\mathrm{M}$ dwarfs. Their relatively nearby distances $(62.23 \pm 0.21 \mathrm{pc}$ and $38.11 \pm 0.23 \mathrm{pc}$, respectively) make them potentially feasible targets for future radial velocity follow-up and atmospheric characterization, although such observations may require substantial investments of time on large telescopes.
\end{abstract}

Unified Astronomy Thesaurus concepts: Mini Neptunes (1063); Exoplanets (498); Transits (1711); M stars (985); Super Earths (1655)

\section{Introduction}

The Transiting Exoplanet Survey Satellite (TESS; Ricker et al. 2015) follows the 8 year missions of Kepler (Borucki

\footnotetext{
31 NSF Graduate Research Fellow.

32 NASA Postdoctoral Program Fellow.

${ }^{33}$ Sagan Fellow.
}

et al. 2010) and K2 (Howell et al. 2014), which discovered thousands of planets. While Kepler typically found planets orbiting faint and distant stars, TESS is examining the brightest and nearest stars for evidence of exoplanet transits. Over the course of its 2 year primary mission, TESS has surveyed $85 \%$ of the sky, looking at over 200,000 nearby stars with a 2 minute cadence and many more stars with the 30 minute full-frame 


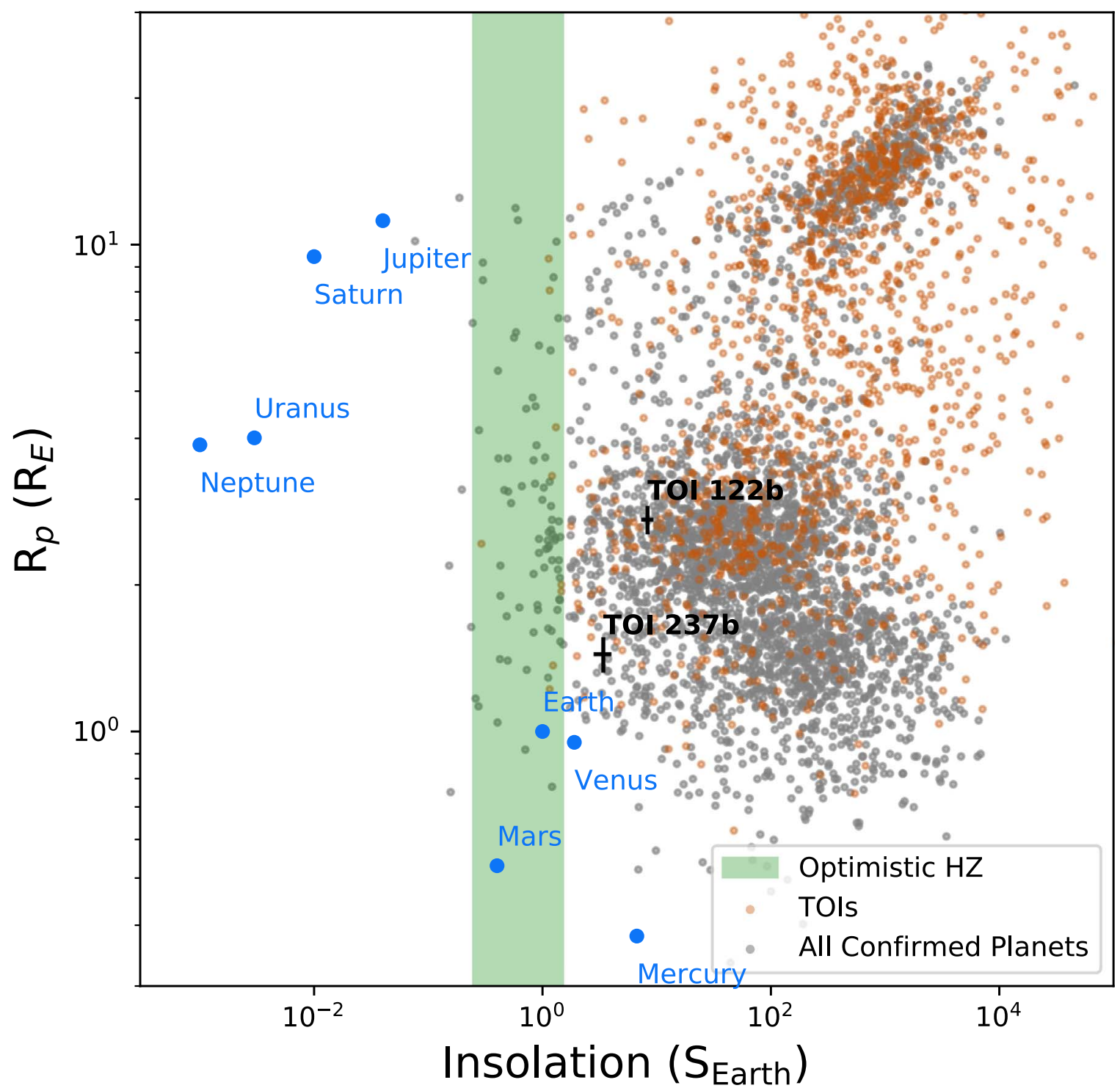

Figure 1. All confirmed exoplanets and current TESS Objects of Interest (TOIs) (as of 2020 February) with current values for $R_{\mathrm{p}}\left(R_{\oplus}\right)$ and $S\left(S_{\oplus}\right)$. Orange points are the TOIs (validated and unvalidated), while the gray points are all confirmed exoplanets (as of 2020 March). Highlighted in green is the "recent Venus-early Mars" habitable zone covering $0.25-1.5 S_{\oplus}$ (e.g., Kopparapu et al. 2019), in which a few systems fall. This optimistic habitable zone is likely shifted to lower insolations for M dwarfs given more recent studies of energy budgets and albedos for M-dwarf planets (Shields et al. 2019).

images (FFIs). TESS is expected to find up to 4500 planets, 500-1200 planets orbiting $\mathrm{M}$ dwarfs, and about 50 planets within 50 pc (see Sullivan et al. 2015; Barclay et al. 2018; Ballard 2019).

M dwarfs are interesting targets for transiting exoplanet studies as they provide the best opportunity for finding temperate terrestrial planets (Blake et al. 2008; Nutzman \& Charbonneau 2008). All main-sequence stars less massive than $0.6 M_{\odot}$ fall into the M-dwarf category, and they are the most numerous stellar type in the universe (e.g., Chabrier \& Baraffe 2000). These stars are very cool $\left(2000 \mathrm{~K}<T_{\text {eff }}<4000 \mathrm{~K}\right)$ and very small, so cool planets have shorter periods, higher transit probabilities, and deeper transits than they would around larger stars.

$\mathrm{M}$ dwarfs tend to host terrestrial exoplanets more often than gas giants (Bowler et al. 2015; Mulders et al. 2015), and these terrestrial planets can more readily be found at lower insolations given the low luminosities of $\mathbf{M}$ dwarfs. Finally, $\mathrm{M}$ dwarfs have such long lifetimes that not a single $\mathrm{M}$ dwarf ever formed has yet evolved off the main sequence (Laughlin et al. 1997), making these stellar systems interesting laboratories for very long timescale planetary evolution. For a comprehensive review of $\mathrm{M}$ dwarfs as exoplanet host stars, see Shields et al. (2019). While the habitability of planets around $\mathrm{M}$ dwarfs remains an open question, the low insolations of $\mathrm{M}$ dwarf planets on short periods creates opportunities for statistically studying the presence and evolution of planetary atmospheres.

The first year of TESS yielded several small exoplanets orbiting M dwarfs such as LHS 3844b (Vanderspek et al. 2019), the L 98-59 system (Cloutier et al. 2019; Kostov et al. 2019), the TOI 270 system (Günther et al. 2019), the Gl 357 system (Luque et al. 2019), LTT 1445Ab (Winters et al. 2019), the LP 791-18 system (Crossfield et al. 2019), and L 168-9b (Astudillo-Defru et al. 2020). The two planets we present in this paper are challenging for precise radial velocity (RV) mass measurements, but both are smaller than $3 R_{\oplus}$ and their low insolations and short periods (see Figure 1) position them as 

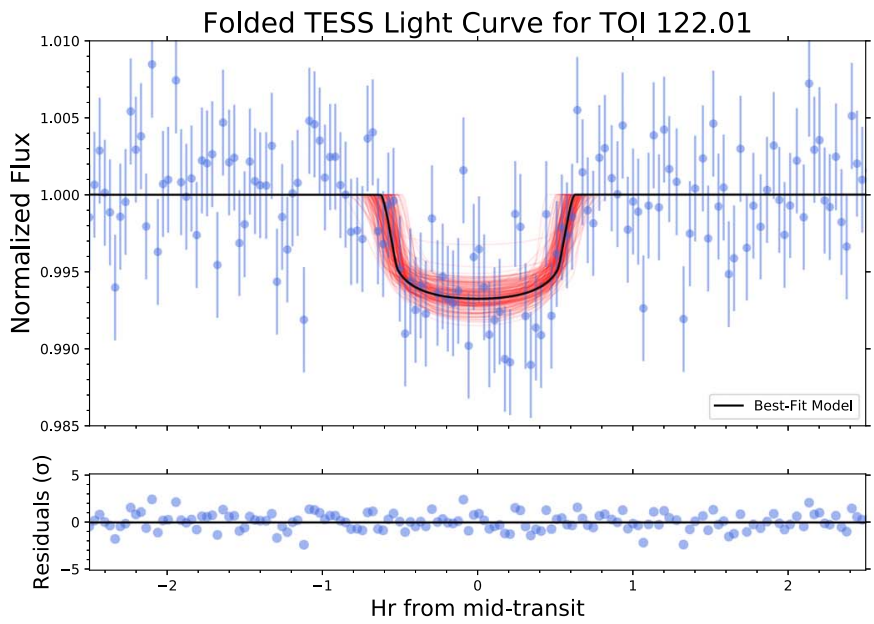

(a)
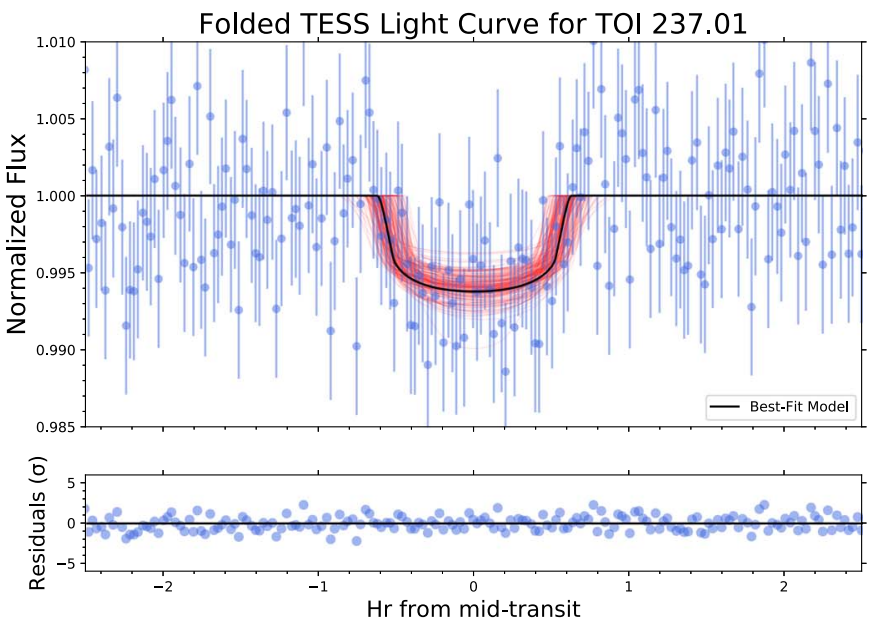

(b)

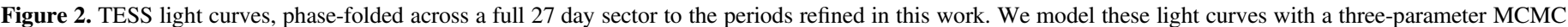

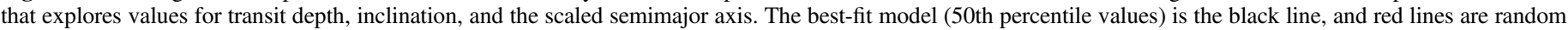

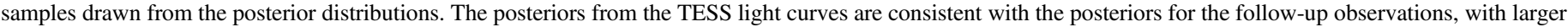
uncertainties. The follow-up observations have allowed us to constrain the transit parameters effectively.

interesting candidates for atmospheric follow-up. They may have retained their atmospheres despite being too hot to be considered habitable and may help us understand atmospheric evolution and the diversity of atmospheres of small planets.

The rest of the paper is organized as follows. In Section 2 we describe the TESS observations, the photometric and spectroscopic follow-up data we gathered, and arguments against these planets being false positives. In Section 3 we describe the results of stellar parameter estimation and transit light-curve fitting, and in Section 4 we discuss the results and their implications for future work.

\section{Data}

\subsection{TESS Photometry}

TESS has four $24^{\circ} \times 24^{\circ}$ field-of-view cameras, each with four $2 \mathrm{k} \times 2 \mathrm{k}$ CCDs. The TESS bandpass is $600-1000 \mathrm{~nm}$, and the pixel scale is $21^{\prime \prime}$ (Ricker et al. 2015). For our analysis of the TESS light curves (Figure 2), we accessed the TESS data using lightkurve (Lightkurve Collaboration et al. 2018) and downloaded the Science Processing Operations Center (SPOC; Jenkins et al. 2016) Presearch Data Conditioning Simple Aperture Photometry (PDCSAP) flux light curves (Smith et al. 2012; Stumpe et al. 2012, 2014). The light curves shown in Figure 2 are 2 minute cadence data phase-folded to the orbital periods we refined in this work.

TOI 122b (TIC 231702397) was observed in Sector 1 of TESS from 2018 July 25 to 2018 August 22 with CCD 1 of Camera 2. Four transits were observed with a 5.1 day period and a 6 ppt depth. The SPOC pipeline flagged the light curve as a planet candidate and it was submitted to the MIT TOI alerts page $^{34}$ (Guerrero et al., submitted), where we accessed the preliminary SPOC data validation transit parameters (Twicken et al. 2018; Li et al. 2019) and scheduled follow-up observations with ground-based observatories. Preliminary parameters indicated that the stellar host was an $\mathrm{M}$ dwarf, implying that the orbiter was super-Earth or sub-Neptune in size.

\footnotetext{
34 https://tess.mit.edu/toi-releases/
}

TOI 237b (TIC 305048087) was observed in Sector 2 of TESS from 2018 August 22 to 2018 September 20 with CCD 1 of Camera 1. Five transits were observed with a 5.4 day period and a 6 ppt depth. The SPOC pipeline flagged the light curve as a planet candidate and it was submitted to the MIT TOI alerts page, where we accessed the preliminary transit parameters and scheduled follow-up observations with ground-based observatories. Preliminary parameters indicated that the stellar host was an $\mathrm{M}$ dwarf, implying that the orbiter was also super-Earth in size.

\subsection{Ground-based Photometry}

The follow-up observations are summarized in Table 1. Both systems were observed extensively as part of the TESS Followup Observing Program Sub-Group 1 (TFOP SG1) photometric campaign. Ground-based observations span several months for both targets, from observatories around the globe. For both TOI 122 and TOI 237, we used the TESS transit finder tool, which is a customized version of the Tapir software package (Jensen 2013), to schedule the photometric time-series observations. Ground-based light curves used in the analysis are shown in Figures 3 and 4.

Las Cumbres Observatory (LCO) photometry. Most photometric data were taken at Las Cumbres Observatory sites via the Las Cumbres Observatory Global Telescope (LCOGT) network (Brown et al. 2013). These observations were done with $1 \mathrm{~m}$ telescopes equipped with Sinistro cameras which have a plate scale of 0 !! 389 and a field of view of $26 ! 4 \times 26 ! 4$. Filters and photometric aperture radii vary between observations and are provided in Table 1. Additional information and the full data sets can be found on ExoFOP-TESS. ${ }^{35}$

LCOGT data are reduced via a standard reduction pipeline (BANZAI; McCully et al. 2018) which performs bias and dark subtractions, flat-field correction, bad pixel masking, astrometric calibration, and source extraction. ${ }^{36}$ We scheduled most observations in red bandpasses $\left(I, i^{\prime}, z\right)$ where the signal-tonoise ratio $(\mathrm{S} / \mathrm{N})$ is highest for $\mathrm{M}$ dwarfs. Observing windows

\footnotetext{
35 https://exofop.ipac.caltech.edu/tess/

36 https://lco.global/documentation/data/BANZAIpipeline/
} 
Table 1

Ground-based Follow-up Observations of the Two Planets, with Mid-transit Times (if a Transit is Detected), Exposure Times, and Filters

\begin{tabular}{|c|c|c|c|c|c|}
\hline Date & Observatory & Filter & Exposure Time (s) & Aperture Radius (") & Transit Midpoint (BJD TDB) \\
\hline \multicolumn{6}{|l|}{ TOI $122 b$} \\
\hline 2018-09-18 & SSO iTelescope & Clear & 120 & 4.8 & (Egress Only) \\
\hline $2018-09-18$ & LCO SSO (1 m) & $r^{\prime}$ & 180 & 4.28 & $2458379.901563_{-0.001189}^{+0.0001239}$ \\
\hline 2018-09-18 & LCO SSO (1 m) & $i^{\prime}$ & 30 & 3.89 & \\
\hline 2018-10-18 & LCO SAAO (1 m) & $I$ & 42 & 5.45 & (Too Noisy) \\
\hline $2018-11-2$ & TRAPPIST South $(0.6 \mathrm{~m})$ & $I+z^{\prime}$ & 60 & 5.2 & $2458425.602564_{-0.000633}^{+0.000630}$ \\
\hline 2018-11-2 & LCO CTIO (1 m) & $I$ & 42 & 4.67 & \\
\hline 2019-07-10 & LCO SAAO (1 m) & $I$ & 50 & 5.06 & $2458674.427546_{-0.000751}^{+0.000773}$ \\
\hline 2019-07-15 & LCO SAAO (1 m) & $I$ & 50 & 3.50 & (Too Noisy) \\
\hline $2019-07-25$ & LCO CTIO (1 m) & $g^{\prime}$ & 240 & 4.67 & $2458689.657695_{-0.001122}^{+0.001223}$ \\
\hline $2019-07-25$ & LCO CTIO (1 m) & $g^{\prime}$ & 240 & 3.89 & \\
\hline 2019-08-4 & LCO CTIO (1 m) & $V$ & 240 & 5.45 & $2458699.817618_{-0.001965}^{+0.001866}$ \\
\hline \multicolumn{6}{|l|}{ TOI $237 b$} \\
\hline $2018-12-16$ & LCO SAAO (1 m) & $i^{\prime}$ & 65 & 4.67 & (Bad Ephemeris) \\
\hline 2019-05-7 & LCO SAAO (1 m) & $i^{\prime}$ & 100 & 3.89 & (Bad Ephemeris) \\
\hline 2019-06-2 & TRAPPIST South $(0.6 \mathrm{~m})$ & $I+z^{\prime}$ & 60 & 5.2 & $2458637.922471_{-0.001352}^{+0.001419}$ \\
\hline 2019-06-14 & LCO CTIO $(1 \mathrm{~m})$ & $I$ & 60 & 6.22 & $2458648.797058_{-0.001739}^{+0.001854}$ \\
\hline 2019-06-19 & LCO CTIO (1 m) & $I$ & 60 & 8.56 & (Too Noisy) \\
\hline $2019-08-2$ & LCO CTIO (1 m) & $I$ & 75 & 5.45 & $2458697.7197997_{-0.000815}^{+0.000796}$ \\
\hline $2019-08-2$ & LCO CTIO (1 m) & $g^{\prime}$ & 300 & 4.67 & \\
\hline $2019-08-13$ & LCO SAAO $(1 \mathrm{~m})$ & $I$ & 70 & 4.67 & $2458708.592274_{-0.001178}^{+0.001521}$ \\
\hline 2019-09-3 & LCO SAAO (1 m) & $I$ & 70 & 5.06 & $2458730.341868_{-0.001559}^{+0.001127}$ \\
\hline
\end{tabular}

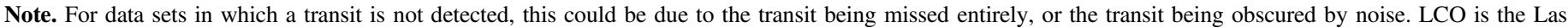

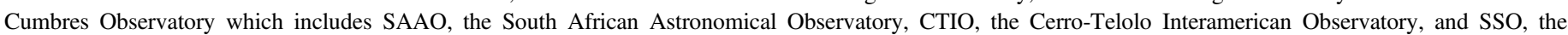

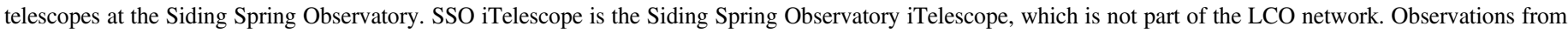

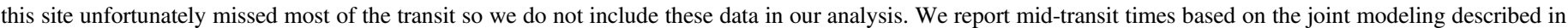
the text.

were chosen to include the full transit along with $1-3 \mathrm{hr}$ of the pre- and post-transit baselines. Many of our observations were defocused, to allow longer integration times for brighter stars and to smear the point-spread function (PSF) over more pixels, reducing any error introduced by uncertainties in the flat field.

We performed differential aperture photometry on the data using the AstroImageJ tool (Collins et al. 2017). Using a finder chart, we drew apertures of varying radii (see Table 1) around the target star, 2-6 bright comparison stars, and any stars of similar brightness within 2!.5. Light curves of the nearby stars were examined for evidence of being eclipsing binaries, variable stars, or the true source of the transit signal in TESS' large pixels. For both of these systems, the transit was found around the target star, and no evidence of nearby eclipsing binaries or periodic stellar variation was found within 2.5 that could have given rise to the transit signal.

TRAPPIST-South photometry. TRAPPIST-South at ESO-La Silla Observatory in Chile is a $60 \mathrm{~cm}$ Ritchey-Chretien telescope, which has a thermoelectrically cooled $2 \mathrm{k} \times 2 \mathrm{k}$ FLI Proline CCD camera with a field of view of $22^{\prime} \times 22^{\prime}$ and pixel scale of $0 . \prime 65$ pixel $^{-1}$ (Jehin et al. 2011; Gillon et al. 2013). We carried out a full-transit observation of TOI 122 on 2019 November 2 with the $I+z$ filter with an exposure time of $60 \mathrm{~s}$. We took 222 images and made use of AstroImageJ to perform aperture photometry, using an aperture radius of 8 pixels (5."2) given the target PSF of 3 ! 7 . We confirmed the event on the target star on time and we cleared all the stars of eclipsing binaries within the 2.5 around the target star. For TOI 237 the observations were carried out on 2019 June 2 with the $I+z$ filter and exposure time of $60 \mathrm{~s}$. We took 207 images and used AstroImageJ to perform the aperture photometry, using an aperture radius of 8 pixels (5!'2) given the target PSF of 4 ". 3 .

\subsection{Southern Astrophysical Research Telescope Speckle Imaging}

High-angular resolution imaging is needed to search for nearby sources not resolved in the seeing-limited ground-based photometry. Nearby sources can contaminate the TESS photometry, resulting in a diluted transit and an underestimated planetary radius. We searched for nearby sources to TOI 122 with the Southern Astrophysical Research (SOAR) telescope speckle imaging (Tokovinin 2018) on 2018 December 21 in the I band, a similar visible bandpass as TESS. Further details of observations from the SOAR TESS survey are available in Ziegler et al. (2020). We detected no nearby stars within $3^{\prime \prime}$ of TOI 122 within the $5 \sigma$ detection sensitivity of the observation, which is plotted along with the speckle autocorrelation function in Figure 5. Companions within 2.5 mag of the target (which could dilute transit depths by $10 \%$ ) are excluded down to separations of about 0 ." 3 .

\subsection{Stellar Spectra}

Magellan spectra. We obtained near-infrared (near-IR) spectra of TOI 122 and TOI 237 on 2018 December 22 with the Folded-port InfraRed Echellete (FIRE) spectrograph (Simcoe et al. 2008). FIRE is hosted on the 6.5 Baade Magellan telescope at LCO. It covers the $0.8-2.5 \mu \mathrm{m}$ band with a spectral resolving power of $R=6000$. Both targets were observed in the ABBA nod patterns using the 0 " 6 slit. 


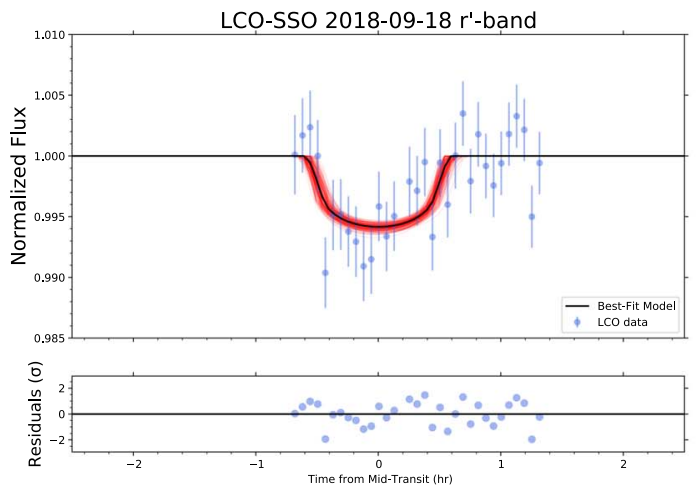

(a)

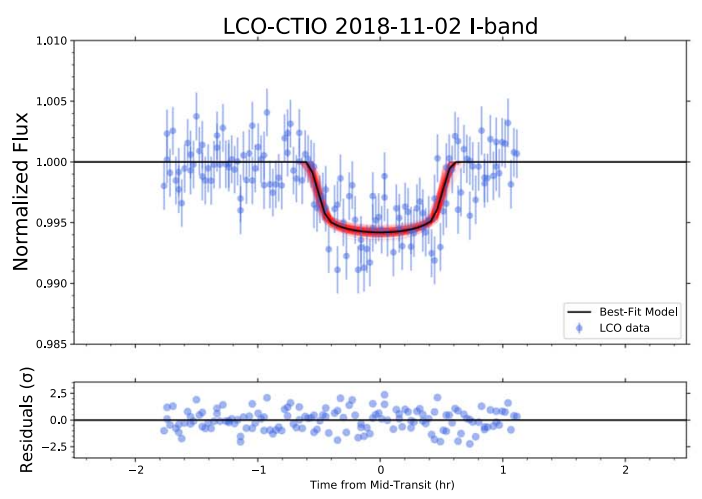

(c)
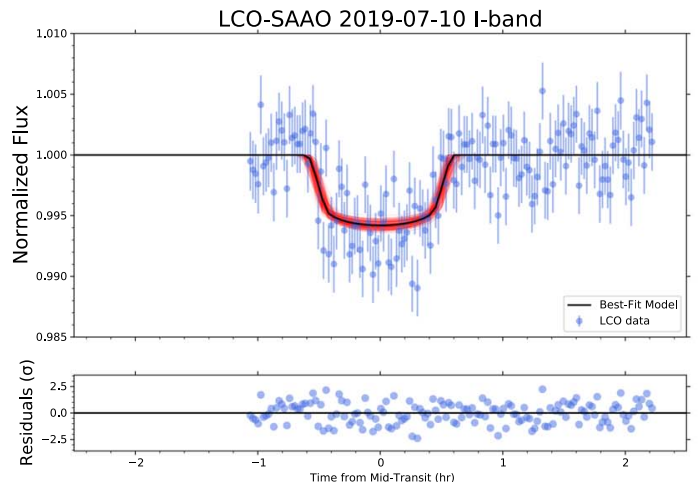

(e)

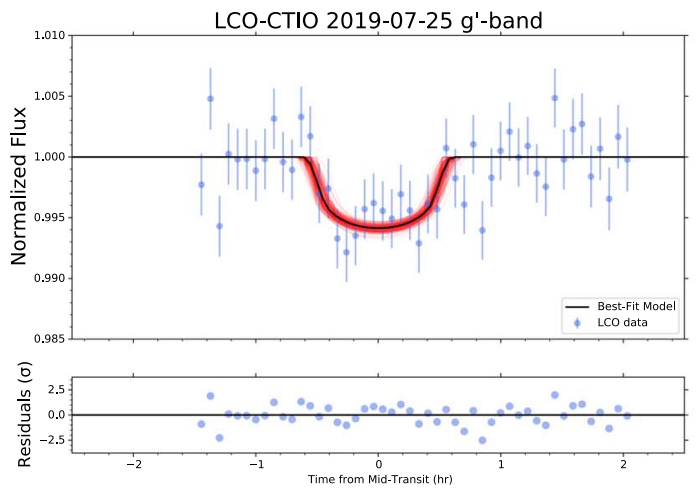

(g)

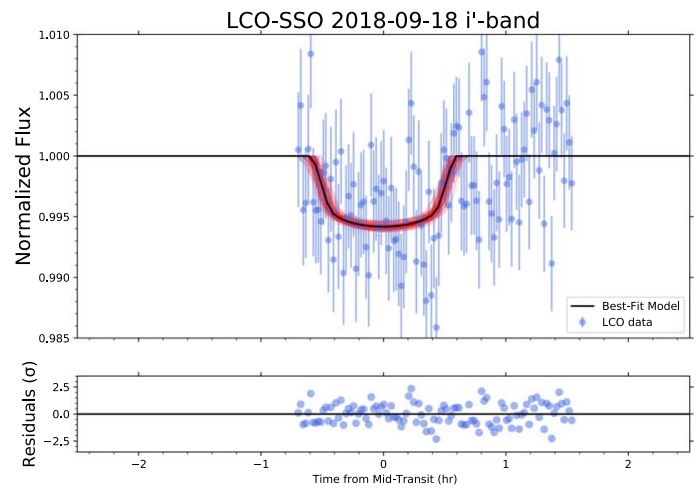

(b)

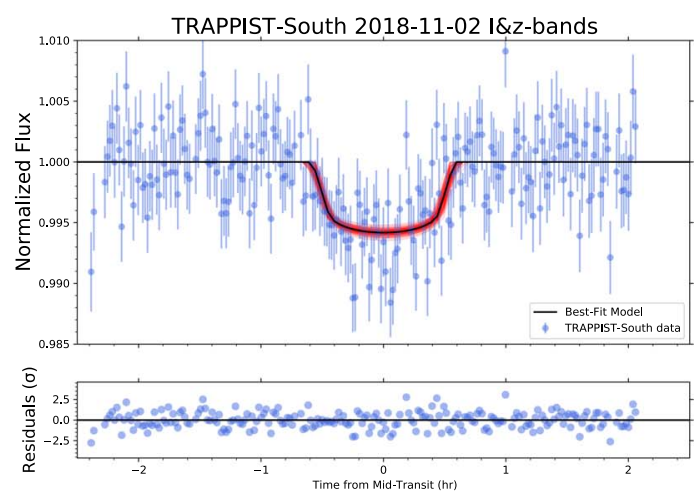

(d)

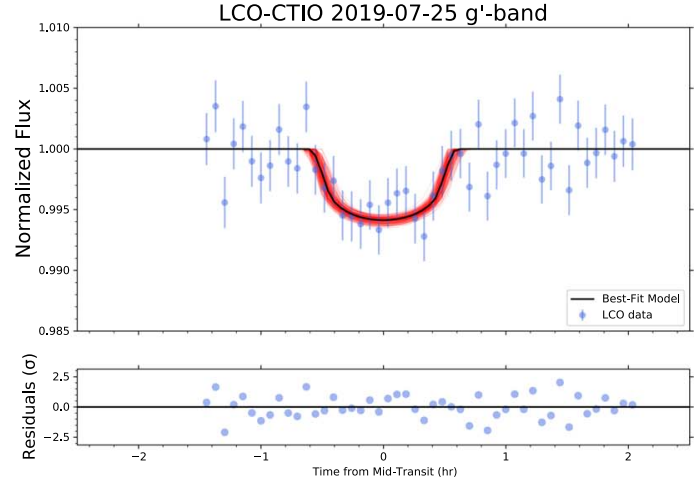

(f)

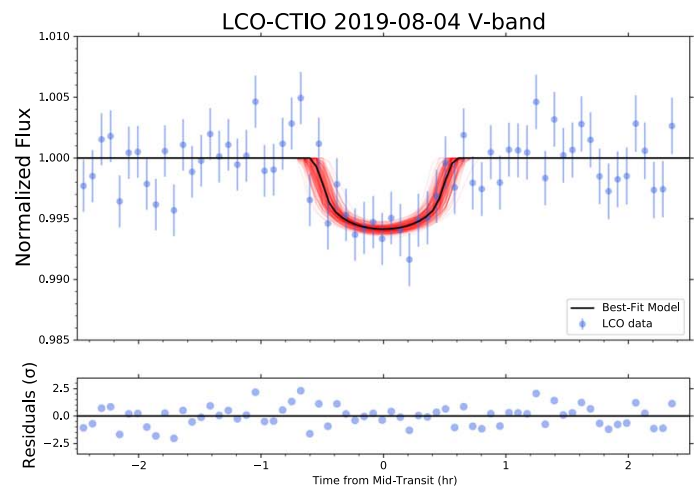

(h)

Figure 3. Light curves for all eight of the viable follow-up transits of TOI 122b. Best-fit MCMC models are in black with 200 random samples plotted in red. Requiring that the transit depth, semimajor axis, and inclination were identical between visits led to a consistent model that fit all the transits. 


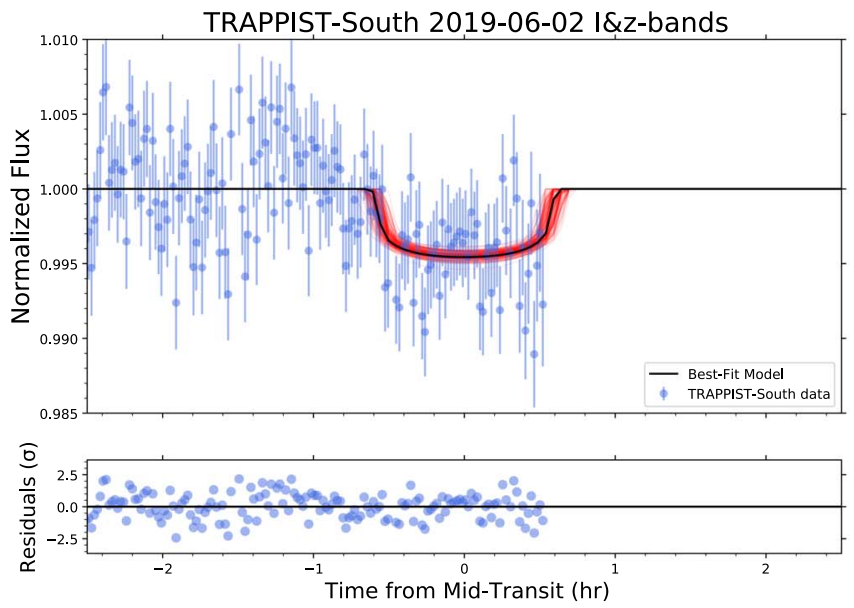

(a)
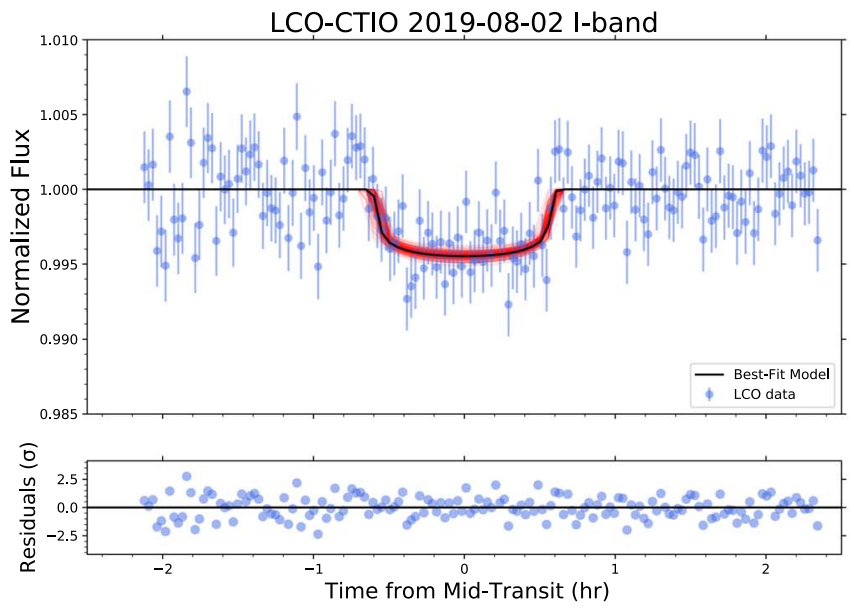

(c)

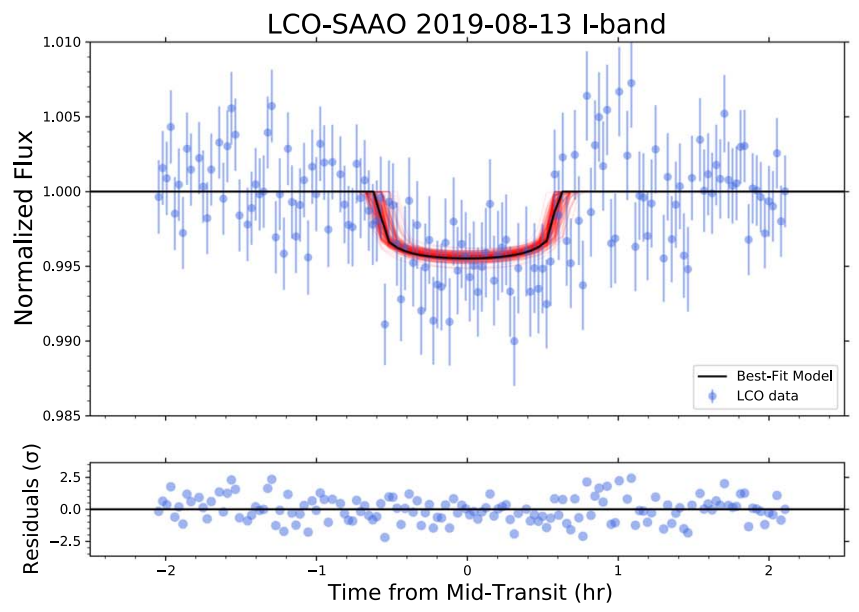

(e)
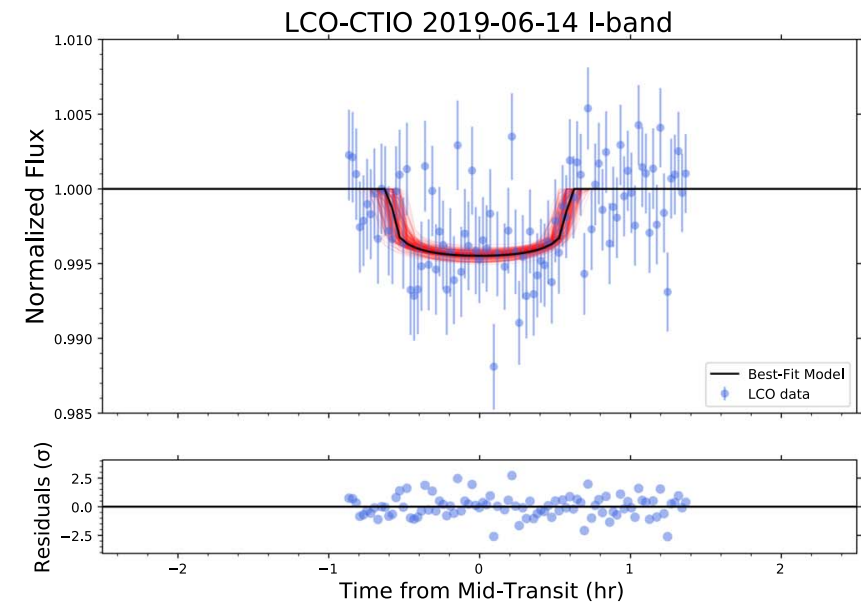

(b)
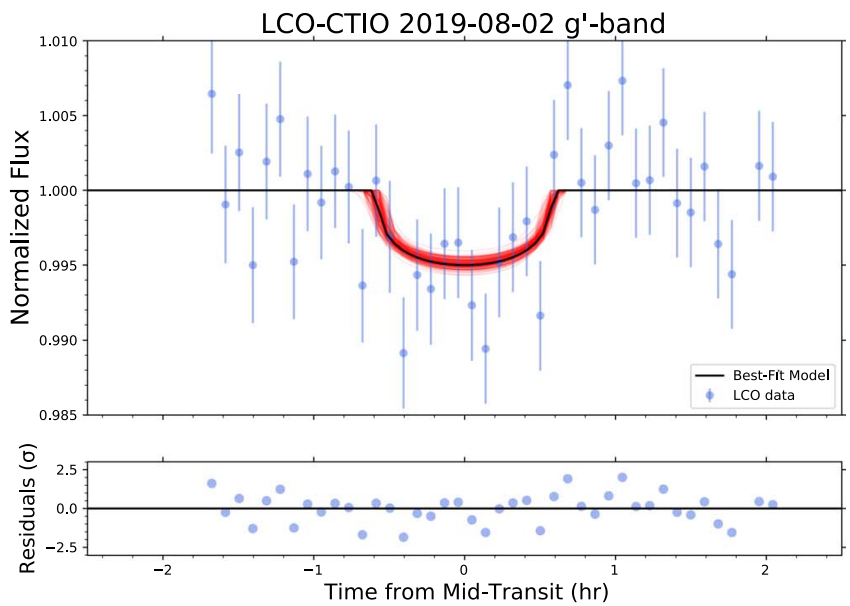

(d)
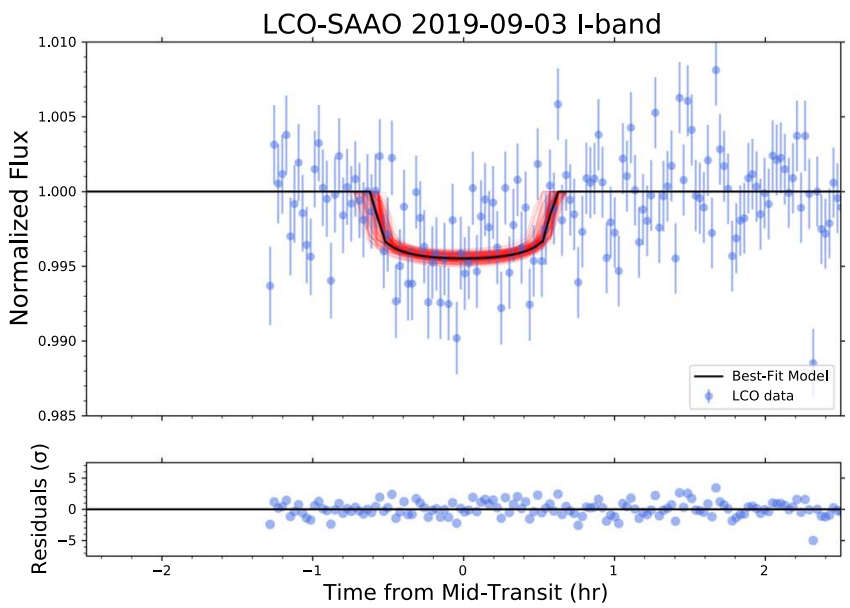

(f)

Figure 4. Light curves for ground-based follow-up transits of TOI 237b. Best-fit MCMC models are in black with 200 random samples plotted in red. Requiring that the transit depth, semimajor axis, and inclination were consistent between visits led to a final model that fit all the transits.

TOI 122 was observed three times and TOI 237 was observed twice, both at a $160 \mathrm{~s}$ integration time. A nearby A0V standard was taken for both targets in order to aid with telluric corrections. The reduction of the spectra were completed using the FIREhose IDL package. ${ }^{37}$

SALT-HRS spectra. We obtained optical echelle spectra for each system using the High-Resolution Spectrograph (HRS;

$\overline{37 \text { http://web.mit.edu/rsimcoe/www/FIRE/ }}$ 


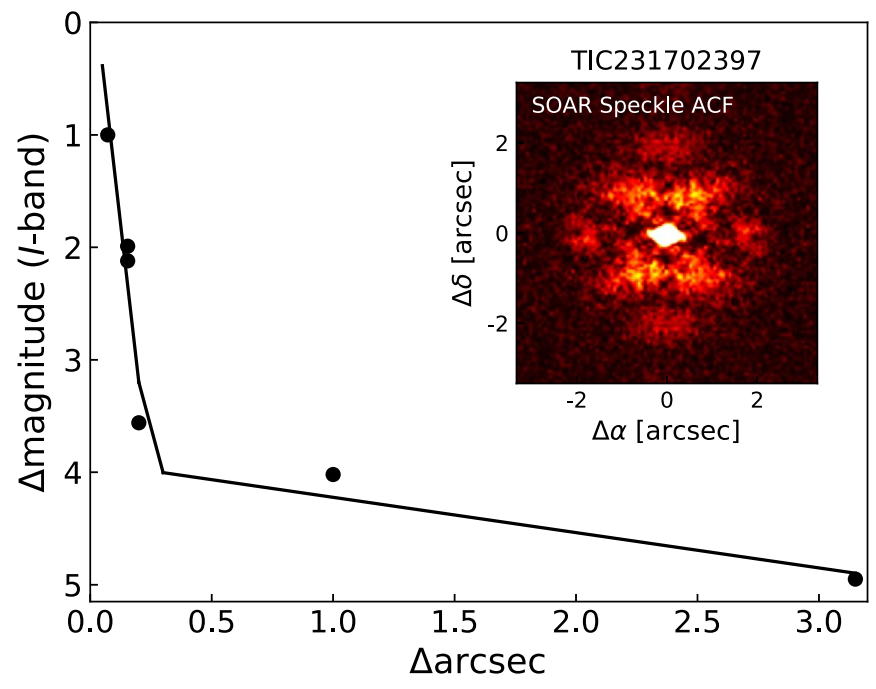

Figure 5. $5 \sigma$ detection limits of SOAR speckle imaging for TOI 122 . The inset shows that no companions were detected down to a limit of $3^{\prime \prime}$.

Crause et al. 2014) on the Southern African Large Telescope (SALT; Buckley et al. 2006). Two observations were made for each system (TOI 122 on 2019 August 9, 10; TOI 237 on 2019 August 10, 12), with each epoch consisting of three consecutive integrations in the high-resolution mode $(R \sim 46,000)$. The spectra were reduced using a HRS-tailored reduction pipeline (Kniazev et al. 2016, 2017), ${ }^{38}$ which performed flat-fielding and wavelength calibration. Due to the faint apparent magnitudes of these systems, we focused our analysis on wavelengths greater than $5000 \AA$, where the spectra had an $\mathrm{S} / \mathrm{N}>10$.

To determine systemic radial velocities for both systems and to search for spatially unresolved stellar companions, we computed spectral-line broadening functions (BFs) for each observation. The BF is computed via a linear inversion of the observed spectrum with a narrow-lined template, and represents a reconstruction of the average photospheric absorptionline profile (Rucinski 1992; Tofflemire et al. 2019). For both systems, the $\mathrm{BF}$ is clearly single peaked, indicating a contribution from only one star. Figure 6 presents a region of the SALT-HRS spectrum for each system with its corresponding template and BF.

For each spectrum, the BFs computed for each echelle order were combined and fit with a Gaussian profile to determine the system's RV. Uncertainties on these measurements were derived from the standard deviation of the line fits for BFs combined from three independent subsets of the echelle orders. The RV for each epoch was then calculated as the errorweighted mean of the three consecutive measurements from each night. More detail on this process can be found in Tofflemire et al. (2019). From the two epochs spaced one to two days apart, we found no evidence for RV variability. The mean and standard error of the RV measurements are provided in Tables 2 and 3.

\section{False-positive Vetting}

Instrumental effects or statistical false positive. From the SPOC data validation reports, the TESS detections are significant with an $\mathrm{S} / \mathrm{N}$ of 8.0 for TOI $122 \mathrm{~b}$ and 9.8 for TOI

\footnotetext{
38 http://www.saao.ac.za/ akniazev/pub/HRS_MIDAS/HRS_pipeline.pdf
}

237b. These are both near the $7 \sigma$ detection significance cutoff (Jenkins 2002), which means that these planets were found near TESS' observational limits of discovery. However, given that we redetected transits of both planets from the ground, with consistent depths and timing, we are confident these detections are in fact robust.

Nearby transit or eclipsing binary. For both of these planets, we searched all nearby ( $<2.5$ radius) stars in the seeing-limited LCO data that were bright enough to have caused the detected transits if blended in the TESS photometry. We found no evidence of sources that were variable or eclipsing on the timescale of these planets' orbital periods. Both of these stars have high proper motions, and examination of archival images indicated that there are no bright stars at the targets' locations (see Figure 7). In addition, we positively detected a transit in the aperture placed around the target star, so we believe these detections are not due to any physically unbound nearby stars.

Contaminated apertures. The photometric apertures we used for the ground-based observations were typically $<6^{\prime \prime}$ (see Table 1), so we can rule out contaminating sources outside that approximate radius from our target stars. In the TESS data, the PDCSAP light curves have already been corrected for contamination of nearby sources present in the TIC, and our higher-resolution ground-based observations show depths consistent with the TESS light curves. SALT spectra show both sources to be single-lined, indicating a lack of evidence for unresolved luminous companions (see Figure 6). We also obtained SOAR speckle imaging of TOI 122 which indicated there was not a nearby companion down to a separation of 0 ". 3 which could contaminate the aperture (see Figure 5).

Nonplanet transiting object. Based on the measured transit depths and inferred stellar parameters, we can constrain both planets to $R_{\mathrm{p}}<0.8 R_{\mathrm{J}}$, which makes them small enough to be in the planet regime (Burrows et al. 2011). We also estimate upper-limit masses from the SALT RV data. Using the two RV data points for each system, we model a range of masses consistent with these values to estimate the upper-limit planet masses. These models were done using a 100k step Markov Chain Monte Carlo (MCMC; 20k step burn-in) with the baseline and planet mass as free parameters, the assumption of circular orbits, and the only constraining prior that the planet mass is non-negative. We find the upper-limit (95th percentile) masses for both of these planets to be in the planetary regime: $M_{\mathrm{p}} \leqslant 6.7 M_{\mathrm{J}}$ for TOI $122 \mathrm{~b}$ and $2.1 M_{\mathrm{J}}$ for TOI $237 \mathrm{~b}$ (see Figure 8). Lastly, we have transit data in multiple bands for both objects, with consistent depths. This achromaticity suggests that these are nonluminous objects such as planets (see Parviainen et al. 2019).

\section{Results \\ 4.1. Light-curve Analysis}

For both systems, we omitted two observations of TOI 122 and one observation of TOI 237 where the transit is completely obscured by the noise. This corresponds to a photometric rms such that the transit $\mathrm{S} / \mathrm{N}$ is $\sim 1$, which we argue is justified given the large number of observations which clearly show a transit (see Table 1). We also omitted observations that did not capture the mid-transit, to prevent the MCMC walkers from running away with obviously incorrect mid-transit times and semimajor axes. We modeled all ground-based light curves simultaneously by requiring the inclination, $a / R_{\star}$, and $R_{\mathrm{p}} / R_{\star}$ to 

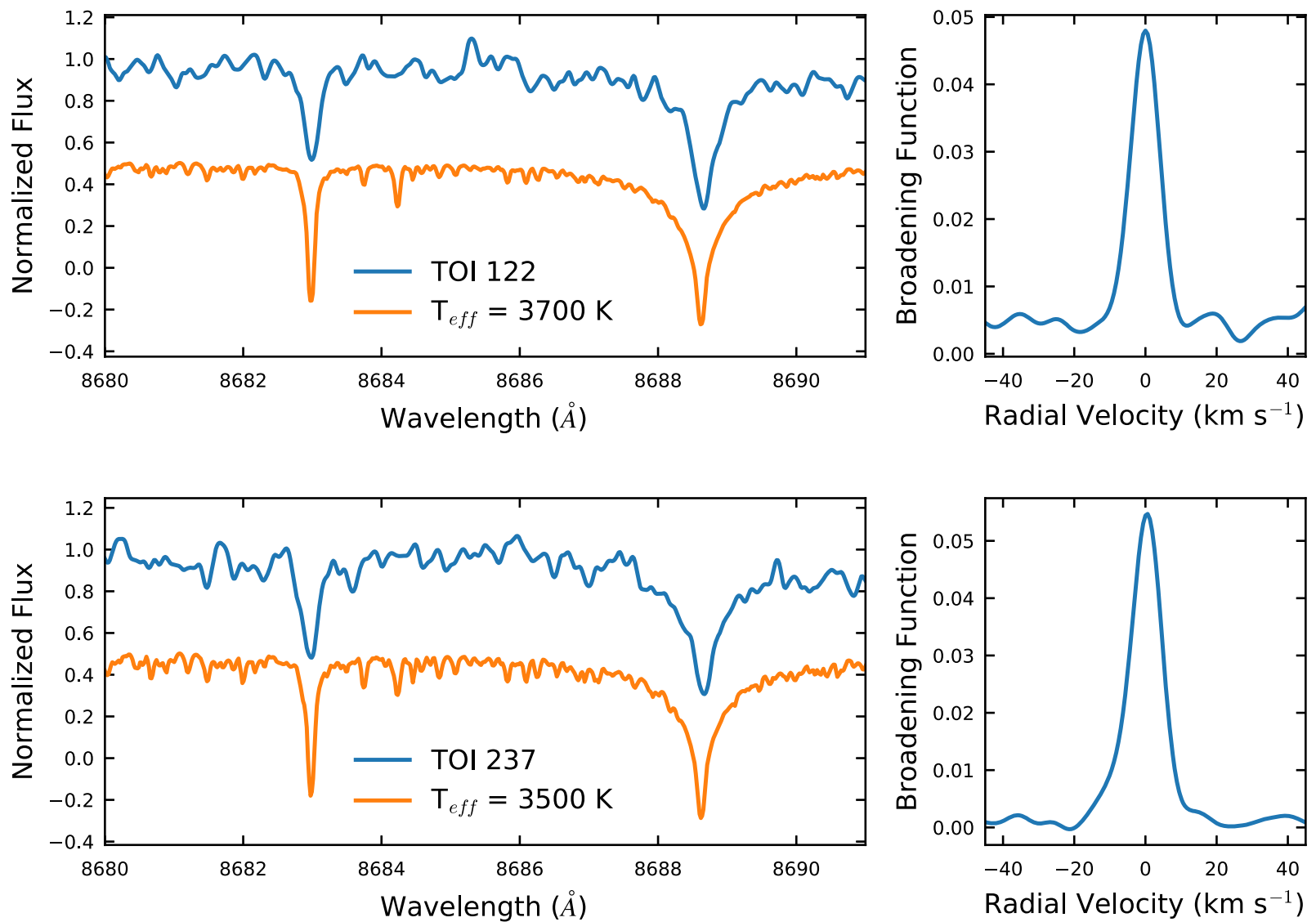

Figure 6. Left: region of a SALT-HRS spectrum (blue) with the corresponding synthetic template (orange), where we have offset the flux slightly for clarity. Right: the $\mathrm{BF}$ computed from this spectral region. Inspection of the BF and individual spectral lines indicates that each system is single lined, and does not host a short-period stellar companion. Note that the model temperatures cited on the figure are higher than the values we report for these two stars; this is discussed in Section 4.2.

be the same value across all transits, but allowing $T_{0}$ to vary for transits at different epochs. $T_{0}$ is fixed between transits that occurred at the same epoch (where we have observations from multiple telescopes, for example). To fit the baseline flux alongside the light-curve parameters, We implemented a linear two-parameter airmass model of the form $\left(C_{1}+C_{2} a\right) B$ where $a$ is the airmass at each exposure and $B$ is the BATMAN lightcurve model. This added up to 24 modeled parameters for TOI $122 \mathrm{~b}$ and 20 parameters for TOI $237 \mathrm{~b}$, the difference being due to a different number of observations for both systems. After analyzing the follow-up light curves and refining the orbital periods, we modeled the phase-folded TESS light curves to examine how well the systems' properties were improved. For a discussion on period refinement, see Section 4.5.

The models are created using BATMAN (Kreidberg 2015), which is based on the analytic transit model from Mandel \& Agol (2002). Stellar limb-darkening coefficients were calculated for each separate bandpass with LDTk, the stellar limbdarkening toolkit (Parviainen \& Aigrain 2015), and these coefficients are listed in Table 4. Figures 2-4 show all transit light curves with models.

We found posterior distributions through Bayesian analysis using emcee (Foreman-Mackey et al. 2013). We ran the MCMC with 150 walkers and 200k steps, discarding the first 40k steps (20\%) and using uniform priors for all parameters. We chose the number of steps based on when each chain converged, using the integrated autocorrelation time heuristic built into emcee. With our 160k steps (post-burn-in), all chains reached $>100$ independent samples, suggesting adequate convergence (for a discussion of MCMC convergence, see Hogg \& Foreman-Mackey 2018). The priors are set so that the planet does not have a negative radius $\left(0 \leqslant R_{\mathrm{p}} / R_{\star} \leqslant 1\right)$, the mid-transit time is within the range of the data, the eccentricity is zero, the semimajor axis is physically reasonable $\left(2 \leqslant a / R_{\star} \leqslant 200\right)$, and the inclination is geometrically limited to be $i \leqslant 90^{\circ}$ to avoid duplicate solutions of $i>90^{\circ}$.

The results cited in Tables 2 and 3 are the 50th percentile values with $1 \sigma$ uncertainties based on the central $68 \%$ confidence intervals of the ground-based MCMC samples which have had the burn-in removed. In Figure 9, we show the posterior distributions from fitting only the folded TESS light curves as well as posterior distributions for only the follow-up transits, for both systems. Results from modeling the follow-up transits are consistent with the TESS fits, but the ground-based follow-up provides much tighter constraints due to the improved $\mathrm{S} / \mathrm{N}$ we get with the larger aperture $\mathrm{LCO} 1 \mathrm{~m}$ telescopes and from having additional independent transits.

\subsection{Stellar Parameters}

Mass and radius. We first used the empirical relations in Mann et al. (2019) to calculate stellar masses from Gaia parallaxes and Two Micron All-Sky Survey (2MASS) $K$-band magnitudes. From Gaia DR2 (Gaia Collaboration et al. 2018), the distance to TOI 122 is $62.23 \pm 0.21 \mathrm{pc}$ and the distance to TOI 237 is $38.11 \pm 0.23$ pc. Using the Mann et al. (2019) relations, we get $M_{\star}=0.312 \pm 0.007 M_{\odot}$ for TOI 122 and 
Table 2

System Parameters for TOI 122b

\begin{tabular}{|c|c|c|}
\hline Parameter & Value & Source \\
\hline \multicolumn{3}{|l|}{ TOI 122} \\
\hline TIC ID & 231702397 & TICv8 \\
\hline R.A. (J2000) & $22: 11: 47.300$ & TICv8 \\
\hline Decl. (J2000) & $-58: 56: 42.25$ & TICv8 \\
\hline TESS Magnitude & $13.048 \pm 0.007$ & TICv8 \\
\hline Apparent $V$ Magnitude & $15.526 \pm 0.026$ & TICv8 \\
\hline Apparent $J$ Magnitude & $11.531 \pm 0.024$ & TICv8 \\
\hline Apparent $H$ Magnitude & $11.020 \pm 0.022$ & TICv8 \\
\hline Apparent $K$ Magnitude & $10.771 \pm 0.021$ & TICv8 \\
\hline Gaia DR2 ID & 6411096106487783296 & Gaia DR2 \\
\hline Distance $(\mathrm{pc})$ & $62.23 \pm 0.21$ & Gaia DR2 \\
\hline $\begin{array}{l}\text { Proper Motion R.A. (mas } \\
\mathrm{yr}^{-1} \text { ) }\end{array}$ & $138.138 \pm 0.089$ & Gaia DR2 \\
\hline $\begin{array}{l}\text { Proper Motion Decl. (mas } \\
\mathrm{yr}^{-1} \text { ) }\end{array}$ & $-235.81 \pm 0.076$ & Gaia DR2 \\
\hline Gaia $G$ mag & 14.3357 & Gaia DR2 \\
\hline Gaia RP mag & 13.1523 & Gaia DR2 \\
\hline Gaia BP mag & 15.7971 & Gaia DR2 \\
\hline Stellar Mass $\left(M_{\odot}\right)$ & $0.312 \pm 0.007$ & $\begin{array}{l}\text { Derived from Mann } \\
\text { et al. (2019) }\end{array}$ \\
\hline Stellar Radius $\left(R_{\odot}\right)$ & $0.334 \pm 0.010$ & $\begin{array}{l}\text { Derived from Mann } \\
\quad \text { et al. (2015) }\end{array}$ \\
\hline$T_{\text {eff }}(\mathrm{K})$ & $3403 \pm 100$ & $\begin{array}{l}\text { Derived from Mann } \\
\text { et al. (2015) }\end{array}$ \\
\hline Luminosity $\left(L_{\odot}\right)$ & $0.0140 \pm 0.0003$ & $\begin{array}{l}\text { Derived from Mann } \\
\text { et al. (2015) }\end{array}$ \\
\hline Stellar $\log g$ & $4.88 \pm 0.05$ & This Work \\
\hline $\mathrm{RV}\left(\mathrm{km} \mathrm{s}^{-1}\right)$ & $-72.4 \pm 1.0$ & This Work \\
\hline Stellar Density $\left(\mathrm{g} \mathrm{cm}^{-3}\right)$ & $12.8_{-4.2}^{+9.5}$ & This Work \\
\hline$v \sin i\left(\mathrm{~km} \mathrm{~s}^{-1}\right)$ & $\leqslant 7.2$ & This Work \\
\hline H $\alpha$ Equivalent Width $(\AA)$ & 0.09 & This Work \\
\hline \multicolumn{3}{|l|}{ TOI $122 b$} \\
\hline Period (days) & $5.078030 \pm 0.000015$ & This Work \\
\hline Transit Depth (\%) & 0.56 & This Work \\
\hline$R_{\mathrm{p}} / R_{\star}$ & $0.075 \pm 0.003$ & This Work \\
\hline Planet Radius $\left(R_{\oplus}\right)$ & $2.72 \pm 0.18$ & This Work \\
\hline Planet Mass $\left(M_{\oplus}\right)$ & $8.8_{-3.1}^{+9.0}$ & $\begin{array}{l}\text { Predicted from Chen } \\
\text { \& Kipping (2017) }\end{array}$ \\
\hline Planet Type & 100\% Neptunian & $\begin{array}{l}\text { Predicted from Chen } \\
\text { \& Kipping (2017) }\end{array}$ \\
\hline$\frac{a}{R_{\star}}$ & $25.2 \pm 1.5$ & This Work \\
\hline Semimajor Axis (au) & $0.0392 \pm 0.0007$ & This Work \\
\hline$i$ (degrees) & $88.4_{-0.4}^{+0.6}$ & This Work \\
\hline Impact Parameter $(b)$ & $0.72_{-0.18}^{+0.07}$ & This Work \\
\hline Insolation $\left(S_{\oplus}\right)$ & $8.8 \pm 1.0$ & This Work \\
\hline $\begin{array}{l}\text { Equilibrium Temperature, } \\
\qquad T_{\mathrm{eq}}(\mathrm{K}) \text { : }\end{array}$ & & This Work \\
\hline $\begin{array}{l}\text { Bond Albedo }=0.75 \\
\quad \text { (Venus-like })\end{array}$ & 333 & \\
\hline $\begin{array}{l}\text { Bond Albedo }=0.3 \\
\quad(\text { Earth-like })\end{array}$ & 431 & \\
\hline $\begin{array}{l}\text { Bond Albedo }=0 \text { (Upper } \\
\text { Limit) }\end{array}$ & 471 & \\
\hline
\end{tabular}

Note. TICv8 information can be found in Stassun et al. (2019).

$M_{\star}=0.179 \pm 0.004 M_{\odot}$ for TOI 237. Using the analogous Mann et al. (2015) absolute $M_{K}$ relation for stellar radii, we found $R_{\star}=0.334 \pm 0.010 R_{\odot}$ and $0.211 \pm 0.006 R_{\odot}$ for TOI 122 and 237 , respectively. As a verification, we compared the stellar densities from the empirical masses and radii to the stellar densities calculated directly from the light curves:

$$
\rho_{\star}=\frac{3 \pi}{G P^{2}}\left(\frac{a}{R_{\star}}\right)^{3},
$$

where $\rho_{\star}$ is the stellar density, $P$ is the orbital period of the planet, $a / R_{\star}$ is the normalized semimajor axis, and we have assumed circular orbits (Seager \& Mallén-Ornelas 2003; Sozzetti et al. 2007). The densities derived from the light curves are $12.8_{-4.2}^{+9.5} \mathrm{~g} \mathrm{~cm}^{-3}$ for TOI 122 and $25.6_{-8.7}^{+4.3} \mathrm{~g} \mathrm{~cm}^{-3}$ for TOI 237, which agree well with the densities from our empirically derived masses and radii $\left(11.8 \pm 2.0 \mathrm{~g} \mathrm{~cm}^{-3}\right.$ and $27.0 \pm 4.0 \mathrm{~g} \mathrm{~cm}^{-3}$ for TOI 122 and 237, respectively). Similarly, we calculated the semimajor axes of these systems from the stellar mass predictions and measured periods, and convert them to $a / R_{\star}$ using the Mann et al. (2015) empirically predicted radii. These calculated semimajor axes give us $a / R_{\star}$ of $25.2 \pm 1.5$ (compared to $25.9_{-3.2}^{+5.3}$ from the light curves) and $34.7 \pm 2.9$ (compared to $34.2_{-4.6}^{+1.9}$ from the light curves) for TOI $122 \mathrm{~b}$ and $237 \mathrm{~b}$.

Effective temperature ( $\left.T_{\mathrm{eff}}\right)$ and luminosity. For both stars, we calculated $T_{\text {eff }}$ using six of the different empirical colormagnitude relations (Equations (1)-(3) and (11)-(13) of Table 2) in Mann et al. (2015). Taking the weighted average of the six temperatures, we get $T_{\text {eff }}=3403 \pm 100 \mathrm{~K}$ for TOI 122 and $3212 \pm 100 \mathrm{~K}$ for TOI 237 . For both sets of calculations, the standard deviation of the six temperatures was $\sim 55 \mathrm{~K}$.

For stellar luminosities, we calculate the $V$-band bolometric correction based on the $V-J$ empirical relation in Mann et al. (2015). This gives luminosities of $0.0140 \pm 0.0003 L_{\odot}$ and $0.0041 \pm 0.0001 L_{\odot}$ for TOI 122 and 237, respectively. We then compared these luminosities to the luminosities calculated from the Mann et al. (2015) radii and effective temperatures (described above):

$$
\frac{L}{L_{\odot}}=\left(\frac{R}{R_{\odot}}\right)^{2}\left(\frac{T_{\mathrm{eff}}}{T_{\odot}}\right)^{4},
$$

where we use $T_{\odot}=5772 \mathrm{~K}$ (Prša et al. 2016). This resulted in $L=0.013 \pm 0.003 L_{\odot} \quad$ for $\quad$ TOI 122 and $L=0.0042 \pm 0.0007 L_{\odot}$ for TOI 237, in good agreement with the bolometric-correction luminosities. Given the collective agreement between light-curve densities, bolometric luminosities, and empirical estimates for radii, masses, and effective temperatures, we adopt the Mann et al. (2015, 2019) derived stellar parameters and corresponding uncertainties for these two stars.

We chose to calculate our stellar parameters based on empirical models rather than adopting values from our spectral observations because of some inconsistencies in the spectra. The method we used to analyze RV signals from SALT spectra is optimized to detect precise RVs but not to accurately calculate stellar temperature. Therefore, the temperature that corresponds to the best-fit RV model is not necessarily an accurate estimate of stellar temperature. This aspect of the modeling does not affect the $v \sin i$ values presented in this paper. The FIRE spectra indicate that TOI 122 is a significantly larger and hotter $\mathrm{M}$ dwarf, opposing other estimates of its size and temperature. We attribute this to the observing conditions and telluric contamination of the Magellan FIRE spectra, and 
Table 3

System Parameters for TOI 237b

\begin{tabular}{|c|c|c|}
\hline Parameter & Value & Source \\
\hline \multicolumn{3}{|l|}{ TOI 237} \\
\hline TIC ID & 305048087 & TICv8 \\
\hline R.A. (J2000) & $23: 32: 58.270$ & TICv8 \\
\hline Decl. (J2000) & $-29: 24: 54.19$ & TICv8 \\
\hline TESS Magnitude & $13.410 \pm 0.007$ & TICv8 \\
\hline Apparent $V$ Magnitude & $16.37 \pm 0.20$ & TICv8 \\
\hline Apparent $J$ Magnitude & $11.74 \pm 0.02$ & TICv8 \\
\hline Apparent $H$ Magnitude & $11.019 \pm 0.022$ & TICv8 \\
\hline Apparent $K$ Magnitude & $10.896 \pm 0.025$ & TICv8 \\
\hline Gaia DR2 ID & 2329387852426700800 & Gaia DR2 \\
\hline Distance (pc) & $38.11 \pm 0.23$ & Gaia DR2 \\
\hline Proper Motion R.A. $\left(\right.$ mas $\mathrm{yr}^{-1}$ ) & $151.047 \pm 0.108$ & Gaia DR2 \\
\hline Proper Motion Decl. $\left(\right.$ mas $\mathrm{yr}^{-1}$ ) & $-333.194 \pm 0.156$ & Gaia DR2 \\
\hline Gaia $G$ mag & 14.754 & Gaia DR2 \\
\hline Gaia RP mag & 13.5016 & Gaia DR2 \\
\hline Gaia BP mag & 16.4447 & Gaia DR2 \\
\hline Stellar Mass $\left(M_{\odot}\right)$ & $0.179 \pm 0.004$ & Derived from Mann et al. (2019) \\
\hline Stellar Radius $\left(R_{\odot}\right)$ & $0.211 \pm 0.006$ & Derived from Mann et al. (2015) \\
\hline$T_{\text {eff }}(\mathrm{K})$ & $3212 \pm 100$ & Derived from Mann et al. (2015) \\
\hline Luminosity $\left(L_{\odot}\right)$ & $0.0041 \pm 0.0001$ & Derived from Mann et al. (2015) \\
\hline Stellar $\log g(\mathrm{cgs})$ & $5.04 \pm 0.07$ & This Work \\
\hline $\mathrm{RV}\left(\mathrm{km} \mathrm{s}^{-1}\right)$ & $7.8 \pm 1.0$ & This Work \\
\hline Stellar Density $\left(\mathrm{g} \mathrm{cm}^{-3}\right)$ & $25.6_{-8.7}^{+4.3}$ & This Work \\
\hline$v \sin i\left(\mathrm{~km} \mathrm{~s}^{-1}\right)$ & $\leqslant 6.4$ & This Work \\
\hline H $\alpha$ Equivalent Width $(\AA)$ & 1.74 & This Work \\
\hline \multicolumn{3}{|l|}{ TOI $237 b$} \\
\hline Period (days) & $5.436098 \pm 0.000039$ & This Work \\
\hline Transit Depth (\%) & 0.38 & This Work \\
\hline$R_{\mathrm{p}} / R_{\star}$ & $0.062 \pm 0.002$ & This Work \\
\hline Planet Radius $\left(R_{\oplus}\right)$ & $1.44 \pm 0.12$ & This Work \\
\hline Planet Mass $\left(M_{\oplus}\right)$ & $3.0_{-1.1}^{+2.0}$ & Predicted from Chen \& Kipping (2017) \\
\hline Planet Type & $25 \%$ Terran, $75 \%$ Neptunian & Predicted from Chen \& Kipping (2017) \\
\hline$\frac{a}{R_{\star}}$ & $34.7 \pm 2.9$ & This Work \\
\hline Semimajor Axis (AU) & $0.0341 \pm 0.0010$ & This Work \\
\hline$i$ (degrees) & $89.5_{-0.6}^{+0.4}$ & This Work \\
\hline Impact Parameter $(b)$ & $0.30_{-0.21}^{+0.27}$ & This Work \\
\hline Insolation $\left(S_{\oplus}\right)$ & $3.7 \pm 0.5$ & This Work \\
\hline Equilibrium Temperature, $T_{\mathrm{eq}}(\mathrm{K})$ : & & This Work \\
\hline Bond Albedo $=0.75$ (Venus-like) & 274 & This Work \\
\hline Bond Albedo $=0.3$ (Earth-like) & 355 & \\
\hline Bond Albedo $=0$ (Upper Limit) & 388 & \\
\hline
\end{tabular}

we therefore do not use the effective temperatures and radii we derive from these spectra.

\subsection{Assumption of Circular Orbits}

All of the analysis was done under the assumption of circular orbits for these two systems. To justify this, we calculate the tidal circularization timescales following Goldreich \& Soter (1966),

$$
\tau_{\text {circ }}=\frac{2 P Q^{\prime}}{63 \pi}\left(\frac{M_{p}}{M_{\star}}\right)\left(\frac{a}{R_{p}}\right)^{5},
$$

where $P$ is the planet's orbital period and $Q^{\prime}$ quantifies how well the planet dissipates energy under deformation. Rocky planets tend to have lower $Q^{\prime}$ values while gaseous planets have larger $Q^{\prime}$ values. We adopt $Q^{\prime}=1 \times 10^{4}$ for TOI $122 \mathrm{~b}$ and $Q^{\prime}=500$ for TOI 237b. These values are based on $Q^{\prime}$ values derived for the solar system planets, where Earth has
$Q^{\prime} \sim 100$ and Neptune has a $Q^{\prime} \sim 6 \times 10^{4}$ (Goldreich \& Soter 1966). We do not have measurements of $M_{p}$ for these planets, but our predicted masses based on the empirical relations in Chen \& Kipping (2017) provide a precise enough estimate for this timescale. For TOI $122 \mathrm{~b}$ and $237 \mathrm{~b}$, we calculate $\tau_{\text {circ }}$ of $0.59 \mathrm{Gyr}$ and $0.17 \mathrm{Gyr}$, respectively.

From the SALT spectra, we derived upper limits on $v \sin i$ to be $<7.2 \mathrm{~km} \mathrm{~s}^{-1}$ for TOI 122 and $<6.4 \mathrm{~km} \mathrm{~s}^{-1}$ for TOI 237 , which allow us to derive lower limits on the rotational periods of both stars under the assumption that the stellar rotation axis is perpendicular to the line of sight. We find those lower limits to be $>2.3$ days for TOI 122 and $>1.7$ days for TOI 237 . In addition, the lack of any significant flaring activity or rotational modulation seen in the TESS light curves for these two systems leads us to assume that the stellar rotational periods are long, and probably greater than 27 days (the TESS observation window for a single sector). While the relation between rotation period and age for $\mathrm{M}$ dwarfs is poorly constrained, Newton 
TOI- $122 \mathrm{~b}=\operatorname{TIC} 231702397 \mid 22^{\mathrm{h}} 11^{\mathrm{m}} 47.7^{\mathrm{s}}-58^{\circ} 56^{\prime} 47.0^{\prime \prime}(2020)$
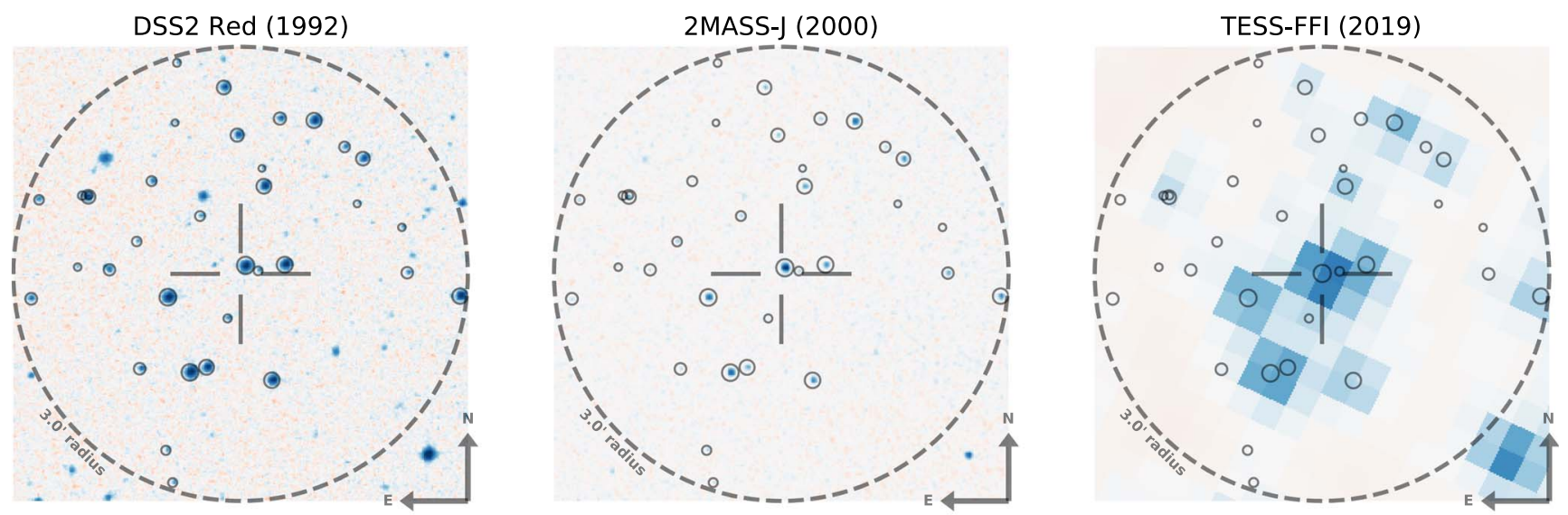

TOI-237b $=\operatorname{TIC} 305048087 \mid 23^{\mathrm{h}} 32^{\mathrm{m}} 58.5^{\mathrm{s}}-29^{\circ} 25^{\prime} 00.9^{\prime \prime}(2020)$
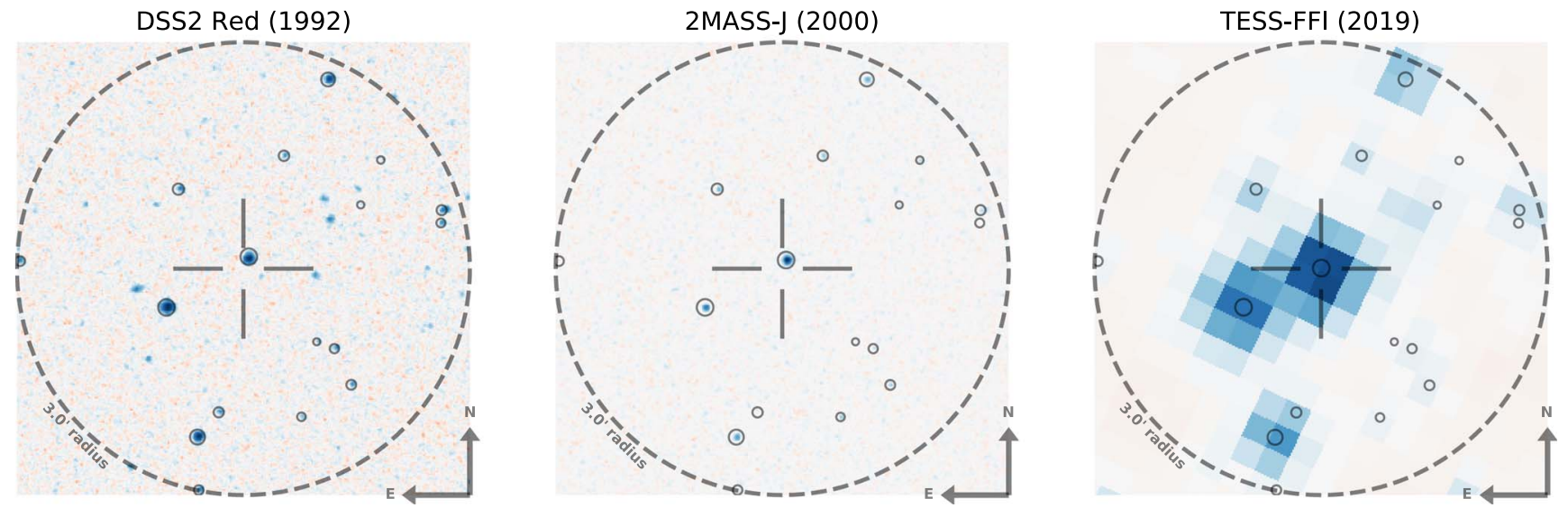

Figure 7. Finder charts for TOI 122 (top) and TOI 237 (bottom), including scanned red-sensitive photograph plates from the Digitized Sky Survey (left), Two Micron All-Sky Survey (middle), and the TESS FFIs (right). Circles indicate stars from Gaia DR2, with areas logarithmically expressing apparent brightness. Crosshairs indicate targets' position in the year 2019, near the time of the TESS imaging.

et al. (2016) found the rotation rates of field $M$ dwarfs to be between 0.1 and 140 days, with $M$ dwarfs younger than $2 \mathrm{Gyr}$ having rotational periods less than 10 days. We also calculate the $\mathrm{H} \alpha$ equivalent widths (EW) from the SALT spectra, as $\mathrm{H} \alpha$ emission is indicative of the activity level of $\mathrm{M}$ dwarfs (see Newton et al. 2017). We find the EWs to be $0.09 \AA$ for TOI 122 and $1.74 \AA$ for TOI 237, placing both of these stars in the canonically inactive regime $(\mathrm{EW}>-1 \AA)$. Newton et al. (2017) provide a more direct way to estimate the rotational periods of inactive $M$ dwarfs based on a polynomial fit with stellar mass. Given our derived masses for these two stars, we predict $\mathrm{P}_{122}=72 \pm 22 \mathrm{~d}$ and $\mathrm{P}_{237}=102 \pm 22 \mathrm{~d}$ from that relation. From the age-inactivity-spectral type relationship for cool stars described in West et al. (2008), we predict that TOI 122 (an M3V) is likely older than 2 Gyr, and TOI 237 (an M4.5V; spectral types based on Rajpurohit et al. 2013) is likely older than $4.5 \mathrm{Gyr}$, consistent with our other estimates of their ages.

We can see a picture emerging that these stars are inactive, slowly rotating, and old, in spite of precise stellar ages being difficult to obtain for $\mathrm{M}$ dwarfs. Given that $\tau_{\text {circ }}$ for both planets is $<1 \mathrm{Gyr}$, we assume both planets are on circular orbits. Our assumption that eccentricity is $\sim 0$ is also supported by the agreement between the stellar densities calculated from the light curves and densities based on empirical estimates of mass and radius (see Section 4.2).

\subsection{Insolation and $\mathrm{T}_{e q}$}

In order to form a picture of the thermal environment of these planets, we calculate the insolation these planets receive, relative to the bolometric flux that Earth receives from the Sun. We also calculate equilibrium temperatures under different assumptions for the Bond albedo, $A_{\mathrm{B}}$, which is the fraction of incident stellar radiation that is reflected by the planet, integrated over both wavelength and angle.

Under the assumptions of circular orbits, efficient heat redistribution, and planets that are thermal emitters (for a discussion of these assumptions, see Cowan \& Agol 2011), we use the $a / R_{\star}$ values derived from our orbital periods and stellar 

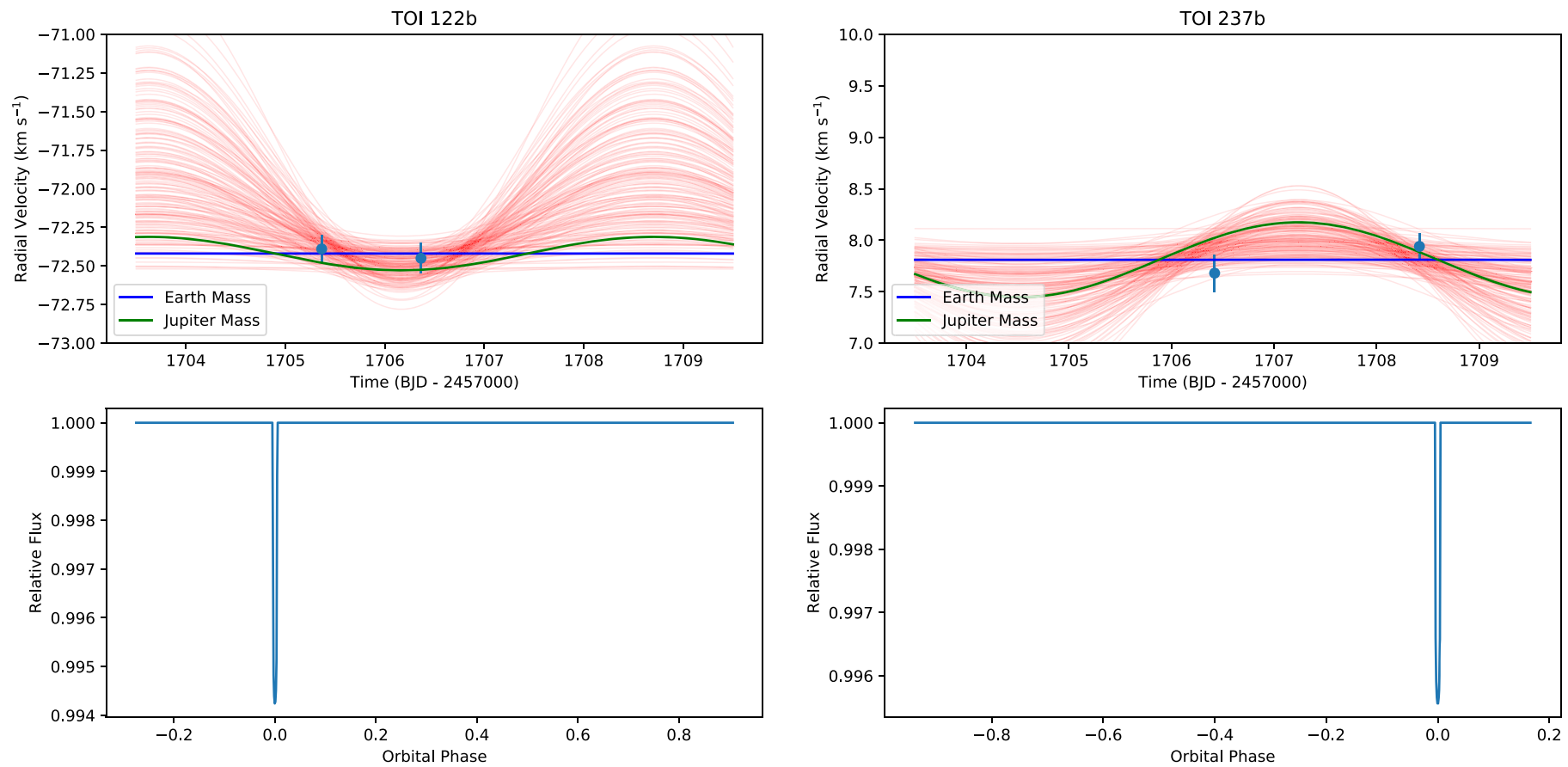

Figure 8. Reconnaissance RV observations from SALT-HRS for both systems, including model orbits for different planet masses (top) plotted with the corresponding transit light curves (bottom). Theoretical RV curves for Earth (blue) and Jupiter (green) masses are shown, as well as 200 random samples from the posterior distributions (red). While we cannot obtain precise planetary masses from these spectra, we are able to rule out super-planetary mass companions by calculating the maximum mass consistent with these measurements. These upper-limit masses based on the 95 th percentile samples are $6.7 M_{\mathrm{J}}$ for TOI $122 \mathrm{~b}$ and $2.1 M_{\mathrm{J}}$ for TOI $237 \mathrm{~b}$.

Table 4

Quadratic Limb-darkening Parameters $\left[u_{1}, u_{2}\right]$ and Associated Uncertainties $\left[\sigma_{1}, \sigma_{2}\right]$, Calculated Using LDTk Using the Stellar Parameters Listed in Tables 2 and 3

\begin{tabular}{lrr}
\hline \hline Filter & Value $\left[u_{1}, u_{2}\right]$ & Uncertainty $\left[\sigma_{1}, \sigma_{2}\right]$ \\
\hline & TOI 122 & \\
$V$ & {$[0.5266,0.2934]$} & {$[0.0151,0.0240]$} \\
$g^{\prime}$ & {$[0.5161,0.2998]$} & {$[0.0124,0.0200]$} \\
$r^{\prime}$ & {$[0.5209,0.2644]$} & {$[0.0149,0.0234]$} \\
$i^{\prime}$ & {$[0.3050,0.2898]$} & {$[0.0069,0.0139]$} \\
$I$ & {$[0.2558,0.2566]$} & {$[0.0046,0.0098]$} \\
$I \& z^{\prime}$ & {$[0.2768,0.2918]$} & {$[0.0067,0.0140]$} \\
\hline & TOI 237 & \\
$g^{\prime}$ & {$[0.5720,0.2925]$} & {$[0.0191,0.0296]$} \\
$I$ & {$[0.2657,0.2911]$} & {$[0.0100,0.0205]$} \\
$I \& z^{\prime}$ & {$[0.2967,0.3343]$} & {$[0.0138,0.0260]$} \\
\hline
\end{tabular}

masses to calculate planetary equilibrium temperature as

$$
T_{\mathrm{eq}}=\left(1-A_{\mathrm{B}}\right)^{\frac{1}{4}}\left(\frac{2 a}{R_{\star}}\right)^{-\frac{1}{2}} T_{\mathrm{eff}},
$$

and insolation as

$$
\frac{S}{S_{\oplus}}=\left(\frac{T_{\mathrm{eff}}}{T_{\odot}}\right)^{4}\left(\frac{a_{\oplus} / R_{\odot}}{a / R_{\star}}\right)^{2},
$$

where $S$ is the bolometric insolation, $a$ is the semimajor axis derived from the stellar masses and orbital periods, $R_{\star}$ is the inferred stellar radius, and $a_{\oplus} / R_{\odot}=215$. We present $T_{\text {eq }}$ (see Tables 2 and 3) as a range of values assuming an Earth-like $A_{\mathrm{B}}=0.3$, a Venus-like $A_{\mathrm{B}}=0.75$, and $A_{\mathrm{B}}=0$.

\subsection{Period Refinement and Transit Timing Variations}

For both systems, we fit a linear model to the TESS epoch and the follow-up epochs to refine the period, which we cite in Tables 2 and 3. In doing this, we are also able to examine the difference between the expected and observed mid-transit times to search for evidence of periodic transit timing variations (TTVs). The reduced $-\chi^{2}$ of a linear ephemeris (2.2 and 2.3 for TOI $122 \mathrm{~b}$ and $237 \mathrm{~b}$, respectively) gave marginal hints of variations on the timescale of minutes, but a Lomb-Scargle periodogram (for a discussion of Lomb-Scargle periodograms, see VanderPlas 2018) applied to the O-C (observed minus calculated) mid-transit times showed no significant periodicity for either system, so we report no significant TTV detection.

\section{Discussion and Conclusions}

These two planets help fill the parameter space for cool worlds near the boundary between rocky and gas-rich compositions. Neither is in the circumstellar habitable zone of its star as both receive more flux than the approximately 0.9 $S_{\oplus}$ moist greenhouse inner-limit calculated by Kopparapu et al. (2013) for stars with these effective temperatures. However, with insolations of $8.8 \pm 1.0$ and $3.7 \pm 0.5 S_{\oplus}$, they are relatively cool among known transiting exoplanets.

\subsection{Radial Velocity Prospects}

We do not have mass-constraining RVs for these two stars, so we applied the Chen \& Kipping (2017) empirical massradius forecaster to predict $M_{122 \mathrm{~b}}=8.8_{-3.1}^{+9.0} M_{\oplus}$ and $M_{237 \mathrm{~b}}=3.0_{-1.1}^{+2.0} M_{\oplus}$, based on the planets' radii. The degeneracy between planet radius and bulk composition leads to large uncertainties in these predicted masses. The forecaster results classify TOI $122 \mathrm{~b}$ as $100 \%$ likely Neptunian and TOI $237 \mathrm{~b}$ as $25 \%$ likely to be Terran and $75 \%$ likely to be 
The Astronomical Journal, 161:13 (17pp), 2021 January
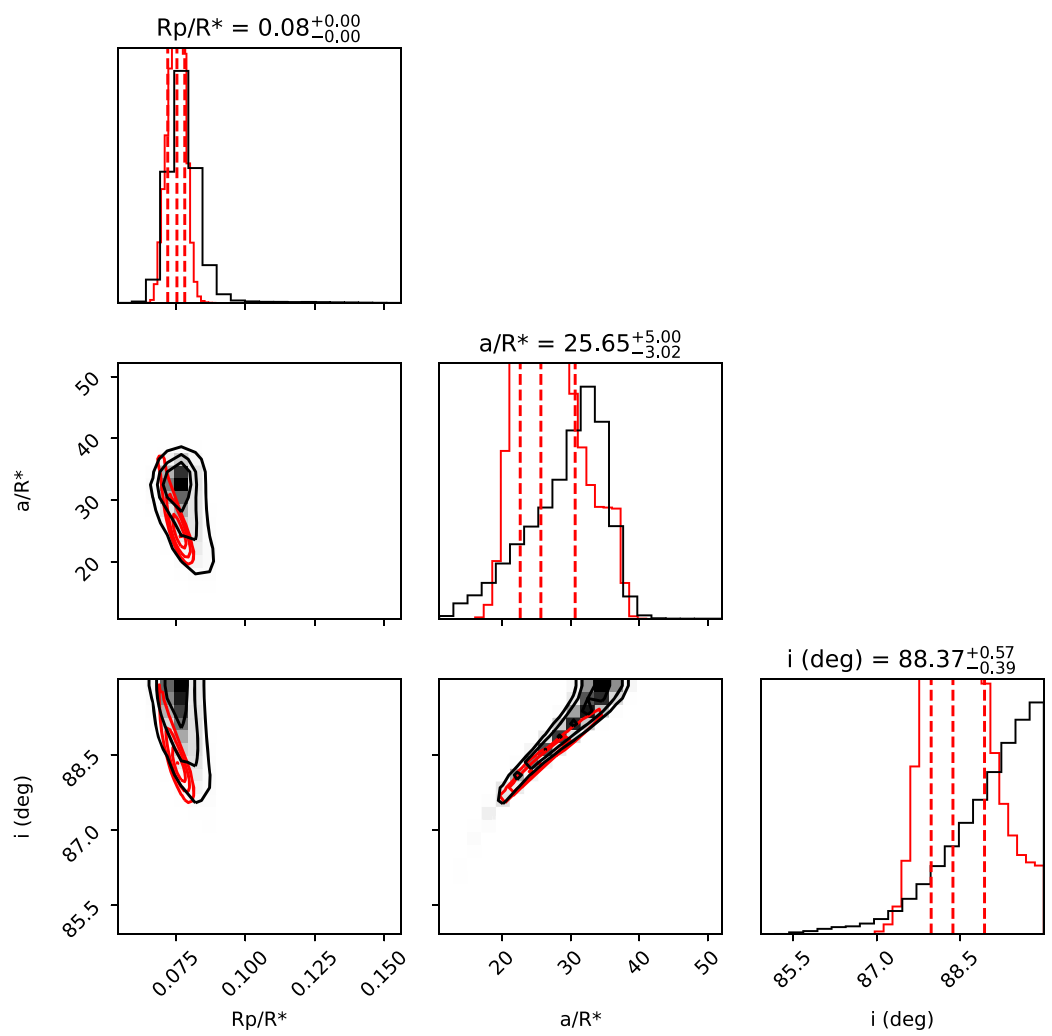

(a)
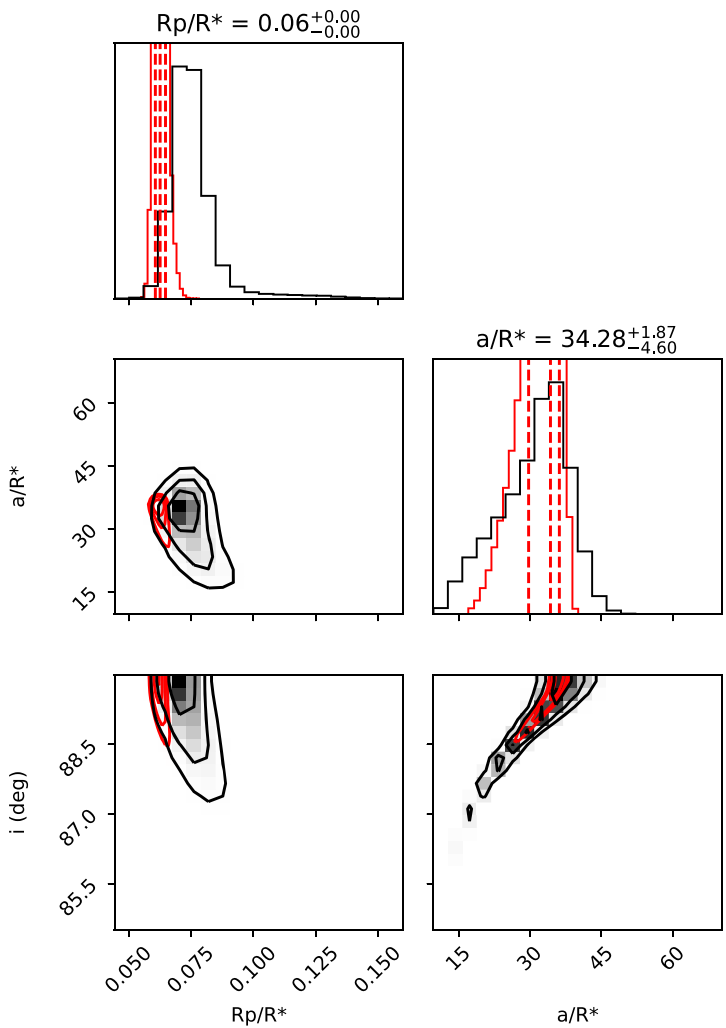

$\mathrm{i}(\mathrm{deg})=89.50_{-0.59}^{+0.35}$

(b)

Figure 9. Corner plots (Foreman-Mackey 2016) for the MCMC posteriors of all fits for TOI 122b (top) and TOI 237b (bottom). The posteriors from modeling only the phase-folded TESS light curves (gray) agree with those from modeling only the ground-based follow-up light curves (black), with the constraints from ground-based telescopes being more precise due to their larger apertures. Labels on top of the posteriors are from the ground-based results. 
Neptunian, where "Terran" is the term used by Chen \& Kipping (2017) to describe worlds similar to the inner terrestrial solar system planets and "Neptunian" is used to describe worlds similar in their basic properties to Neptune and Uranus. The transition between these planet types was found by Chen \& Kipping (2017) to be at $2.0 \pm 0.7 M_{\oplus}$. We can compare the stellar magnitudes and predicted RV semiamplitudes to the current and near-future capabilities of RV facilities. Using the periods, stellar masses, and predicted planet masses, we estimate RV semi-amplitudes of $7.1 \mathrm{~m} \mathrm{~s}^{-1}$ and $3.4 \mathrm{~m} \mathrm{~s}^{-1}$ for TOI $122 \mathrm{~b}$ and $237 \mathrm{~b}$, respectively. These semi-amplitudes are above the instrumental noise floors for many RV spectrographs, although the faint magnitudes of these stars implies that mass-constraining RV measurements will be very time intensive.

The Calar Alto high-Resolution search for M dwarfs with Exoearths with Near-infrared and optical Echelle Spectrographs (CARMENES; Quirrenbach et al. 2010) instrument would require $460 \mathrm{~s}$ exposures to obtain $7.1 \mathrm{~m} \mathrm{~s}^{-1}$ precision for TOI 122 and $2250 \mathrm{~s}$ exposures to obtain $3.4 \mathrm{~m} \mathrm{~s}^{-1}$ precision for TOI $237 \mathrm{~b} .{ }^{39}$ The latter is just beyond the $1800 \mathrm{~s}$ maximum individual exposure time for this instrument, but the former implies the mass of TOI 122b could be within reach of a reasonably ambitious CARMENES observing program. Likewise, the Habitable Zone Planet Finder (HPF) spectrograph (Mahadevan et al. 2012, 2014) could possibly achieve precision as good as $10 \mathrm{~m} \mathrm{~s}^{-1}$ for TOI 122 and $5 \mathrm{~m} \mathrm{~s}^{-1}$ for TOI 237 with 15 minute exposures (see Figure 2 of Mahadevan et al. 2012). With slightly longer exposure times, this instrument may be able to achieve mass-constraining precision for these two planets. The recent discovery of the $G$ 9-40 system (Stefansson et al. 2020) used HPF to constrain planetary masses, achieving $6.49 \mathrm{~m} \mathrm{~s}^{-1}$ precision with exposure times of $945 \mathrm{~s}$. This star has $K_{\mathrm{s}}=9.2$, so scaled to the magnitudes of TOIs 122 and 237, we would need exposure times of $\sim 4 \mathrm{ks}$ to achieve this precision for the systems presented here. Another instrument, the InfraRed Doppler (IRD) for the Subaru telescope (Kotani et al. 2014) also provides some hope. The sensitivity estimator ${ }^{40}$ implies that for both of these stars, $\sim 2 \mathrm{~m} \mathrm{~s}^{-1}$ precision $(\mathrm{S} / \mathrm{N}>100)$ may be possible with $1 \mathrm{hr}$ exposures.

\subsection{Atmospheric Characterization Prospects}

In order to assess the viability of TOI $122 \mathrm{~b}$ and TOI $237 \mathrm{~b}$ for atmospheric studies, we calculated their emission spectroscopy metrics (ESM) following Kempton et al. (2018). This metric represents the $\mathrm{S} / \mathrm{N}$ of a single secondary eclipse observed by the Mid-Infrared Instrument (MIRI) low-resolution spectrometer (LRS) of the James Webb Space Telescope (JWST). The emission $\mathrm{S} / \mathrm{N}$ scales directly as the flux of the planet and the square root of the number of detected photons, and inversely to the flux of the star, so hot planets orbiting cool nearby stars will have a larger ESM.

We calculate the ESM assuming that the planet dayside temperatures are equal to $1.1 \times T_{\text {eq }}$ (following the process outlined in Kempton et al. 2018) and that both have an Earthlike albedo of 0.3 . We find the ESM to be 2.9 for TOI $122 \mathrm{~b}$ and 0.6 for TOI 237b. Compared to GJ $1132 \mathrm{~b}(\mathrm{ESM}=7.5)$ these planets are much less favorable for atmospheric follow-up with

\footnotetext{
39 https://carmenes.caha.es/ext/instrument/index.html

${ }^{40}$ http://ird.mtk.nao.ac.jp/IRDpub/sensitivity/sensitivity.html
}

JWST. A minimum of 12 eclipses would be necessary to achieve an $\mathrm{S} / \mathrm{N}>10$ for TOI $122 \mathrm{~b}$ and a minimum of 278 eclipses would be needed for TOI $237 \mathrm{~b}$, as the $\mathrm{S} / \mathrm{N}$ scales as $\sqrt{N_{\text {obs }}}$. Detecting thermal emission with JWST would be challenging for TOI 122b and impractical for TOI 237b.

We also calculate the transmission spectroscopy metric (TSM) from Kempton et al. (2018). This metric corresponds to the expected $\mathrm{S} / \mathrm{N}$ of transmission features for a cloud-free atmosphere, over $10 \mathrm{hr}$ of observation ( $5 \mathrm{hr}$ in-transit). Our predicted TSMs are 54 for TOI $122 \mathrm{~b}$ and 7 for TOI $237 \mathrm{~b}$, which imply that these planets could both be amenable to transmission spectroscopy with the Near-Infrared Imager and Slitless Spectrograph (NIRISS) of JWST, although planetary mass measurements would be necessary to make precise inferences from their transmission spectra (Batalha et al. 2019).

\subsection{Volatile Evolution}

These two planets span an interesting range of radii and insolations, making them exciting cases that may help us learn more about the diversity of atmospheres possessed by small planets orbiting $\mathrm{M}$ dwarfs. Figure 10 shows the Jeans escape parameter (e.g., Ingersoll 2013, Box 2.2) for these systems as well as solar system bodies and all confirmed exoplanets for which this parameter could be calculated. This ratio of gravitational-to-thermal energy is an extremely approximate tracer of atmospheric escape, but it can help us qualitatively understand the relative susceptibility of different planets to atmospheric loss. With only loose predictions for the masses of TOI $122 \mathrm{~b}$ and TOI $237 \mathrm{~b}$, their position on this plot leaves us with an ambiguous picture of whether they have atmospheres and what their compositions could be. They may even represent the transition between worlds that have lost almost all of their $\mathrm{H} / \mathrm{He}$ (such as Earth and Venus) and worlds that have retained those lighter elements (such as Neptune or Uranus). Though we cannot determine any strong constraints with this Jeans approximation alone, these two planets are not in a regime where they would have obviously lost their atmospheres, as Mercury and Mars have. A more detailed investigation into the current and past XUV irradiation, which is a main driver of atmospheric loss, would be necessary to more cleanly place these planets in context (Zahnle \& Catling 2017).

TOI $122 \mathrm{~b}$ is a sub-Neptune-sized planet orbiting an M dwarf that is $33 \%$ the radius of our Sun. It likely has a thick atmosphere but on a 5.1 day orbit, it is far interior to the habitable zone of its star and irradiated at over eight times the flux of Earth. It is dim enough to present a challenge for most existing RV instruments, but mass measurements might be possible with a sufficient investment of time on infrared spectrographs. Its atmosphere is on the edge of detectability in both emission and transmission with JWST. With a relatively low equilibrium temperature, there could be very interesting atmospheric chemistry in this planet's atmosphere that might be observable with sufficiently ambitious observing programs.

TOI $237 \mathrm{~b}$ is a super-Earth-sized planet orbiting a M dwarf that is $21 \%$ the radius of our Sun and only $3200 \mathrm{~K}$. With its 5.4 day orbit, it receives nearly four times Earth insolation from its host star. Given the size of this planet and dimness of the star, mass measurements are likely very difficult to achieve, and we may not know its mass for some time. Even cooler than TOI $122 \mathrm{~b}$, this planet cannot be studied with emission spectroscopy, but transmission spectroscopy is possible and 


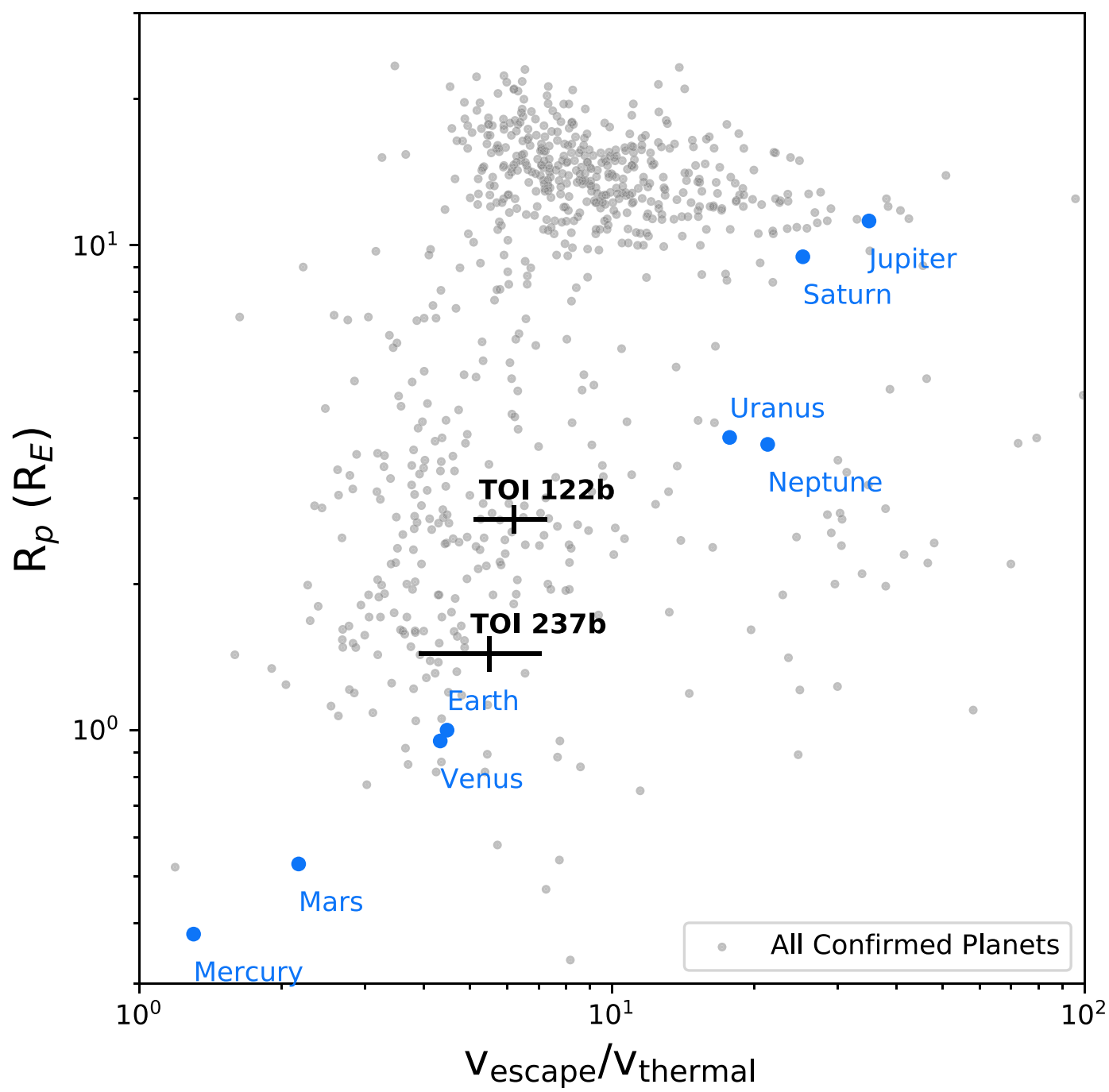

Figure 10. The ratio of planetary escape velocity to the thermal energy of an $\mathrm{H}$ atom at the planetary equilibrium temperature (the "escape parameter"; Jeans 1905 ), for known transiting and solar system planets. TOI $122 \mathrm{~b}$ and TOI $237 \mathrm{~b}$ are included, using predicted masses from Chen \& Kipping (2017) to calculate their gravity. This extremely rough proxy for susceptibility to atmospheric escape indicates these planets may be broadly similar to Earth and Venus, in terms of ongoing mass loss from their atmospheres. This qualitative comparison does not account for the important XUV radiation illuminating the planets, either now or in the past. We estimate the uncertainties for TOI $122 \mathrm{~b}$ and $237 \mathrm{~b}$ by propagating our uncertainties from the planet parameters, which are dominated by large uncertainties on predicted masses.

we may be able to learn about this planet's atmosphere, if it has retained one.

We are left with the following pictures of these systems: TOI $122 \mathrm{~b}$ and TOI $237 \mathrm{~b}$ are two worlds that span planetary radii not seen in our own solar system and are interesting laboratories to study planet formation, dynamics, and composition. Their long periods leave them too cool for emission spectroscopy but as a result, they occupy a very interesting space of relatively cool, though still uninhabitably warm, planets. Thus, they may give us insight to an as-yet poorly understood type of planetary atmosphere. While more targeted atmospheric or RV studies would require a significant investment of time for these two systems, they are valuable additions to the statistical distribution of known planets.

Software Python code used in this paper is available on the author's Github. ${ }^{41}$ This project made use of many publicly available tools and packages for which the authors are immensely grateful. In addition to the software cited throughout the paper, we also used Astropy (Astropy Collaboration

$\overline{41}$ https://github.com/will-waalkes/TOI237and122 et al. 2013), NumPy (van der Walt et al. 2011), Matplotlib (Hunter 2007), Pandas (McKinney 2011), and Anaconda's JupyterLab.

Funding for the TESS mission is provided by NASA's Science Mission directorate. We acknowledge the use of public TESS Alert data from pipelines at the TESS Science Office and at the TESS Science Processing Operations Center. This research has made use of the ExoFOP-TESS website, which is operated by the California Institute of Technology, under contract with the National Aeronautics and Space Administration under the Exoplanet Exploration Program. This paper includes data collected by the TESS mission, which are publicly available from the Mikulski Archive for Space Telescopes (MAST). This material is based upon work supported by the National Science Foundation Graduate Research Fellowship Program under grant No. (DGE1650115) and (DGE-1746045). Any opinions, findings, and conclusions or recommendations expressed in this material are those of the authors and do not necessarily reflect the views of the National Science Foundation. This work makes use of 
observations from the LCOGT network. Resources supporting this work were provided by the NASA High-End Computing (HEC) Program through the NASA Advanced Supercomputing (NAS) Division at Ames Research Center for the production of the SPOC data products. The research leading to these results has received funding from the ARC grant for Concerted Research Actions, financed by the Wallonia-Brussels Federation. TRAPPIST is funded by the Belgian Fund for Scientific Research (Fond National de la Recherche Scientifique, FNRS) under the grant FRFC 2.5.594.09.F, with the participation of the Swiss National Science Fundation (SNF). M.G. and E.J. are F.R.S.-FNRS Senior Research Associates. B.R-A. acknowledges the funding support from FONDECYT through grant 11181295. Z.K.B.T. gratefully acknowledges support for this work from the TESS Guest Investigator program (Award \#80NSSC18K1588) and the National Science Foundation CAREER program (Award \#\#1945633).

\section{ORCID iDs}

William C. Waalkes (1) https://orcid.org/0000-00028961-0352

Zachory K. Berta-Thompson (ㄷ) https://orcid.org/0000-00023321-4924

Karen A. Collins (ㄱ) https://orcid.org/0000-0001-6588-9574

Adina D. Feinstein (1) https://orcid.org/0000-0002-9464-8101

Benjamin M. Tofflemire (10) https://orcid.org/0000-00032053-0749

Bárbara Rojas-Ayala @ $\odot$ https://orcid.org/0000-0002-

0149-1302

Michele L. Silverstein (10 https://orcid.org/0000-00032565-7909

Elisabeth Newton (10) https://orcid.org/0000-0003-4150-841X George R. Ricker (1) https://orcid.org/0000-0003-2058-6662

Roland Vanderspek (ib https://orcid.org/0000-0001-6763-6562

David W. Latham (1) https://orcid.org/0000-0001-9911-7388

S. Seager (1) https://orcid.org/0000-0002-6892-6948

Joshua N. Winn (10 https://orcid.org/0000-0002-4265-047X

Jon M. Jenkins (1) https://orcid.org/0000-0002-4715-9460

Jessie Christiansen (1) https://orcid.org/0000-0002-8035-4778

Robert F. Goeke (1) https://orcid.org/0000-0003-1748-5975

Alan M. Levine (다 https://orcid.org/0000-0001-8172-0453

H. P. Osborn (1) https://orcid.org/0000-0002-4047-4724

S. A. Rinehart (1) https://orcid.org/0000-0003-2519-3251

Mark E. Rose (16) https://orcid.org/0000-0003-4724-745X

Eric B. Ting (1) https://orcid.org/0000-0002-8219-9505

Joseph D. Twicken (1) https://orcid.org/0000-0002-6778-7552

Khalid Barkaoui (1) https://orcid.org/0000-0003-1464-9276

Jacob L. Bean (1) https://orcid.org/0000-0003-4733-6532

César Briceño (1) https://orcid.org/0000-0001-7124-4094

David R. Ciardi (i) https://orcid.org/0000-0002-5741-3047

Kevin I. Collins (1) https://orcid.org/0000-0003-2781-3207

Dennis Conti $\mathbb{1}$ https://orcid.org/0000-0003-2239-0567

Tianjun Gan (1) https://orcid.org/0000-0002-4503-9705

Michaël Gillon (1) https://orcid.org/0000-0003-1462-7739

Emmanuël Jehin (ㄴ) https://orcid.org/0000-0001-8923-488X

Eric L. N. Jensen (1) https://orcid.org/0000-0002-4625-7333

John F. Kielkopf (10) https://orcid.org/0000-0003-0497-2651

Nicholas Law (1) https://orcid.org/0000-0001-9380-6457

Andrew W. Mann (1) https://orcid.org/0000-0003-3654-1602

Benjamin T. Montet (1) https://orcid.org/0000-0001-

7516-8308
Francisco J. Pozuelos (10 https://orcid.org/0000-00031572-7707

Jessica E. Libby-Roberts (1) https://orcid.org/0000-00022990-7613

Carl Ziegler (1) https://orcid.org/0000-0002-0619-7639

\section{References}

Astropy Collaboration, Robitaille, T. P., Tollerud, E. J., et al. 2013, A\&A, 558, A33

Astudillo-Defru, N., Cloutier, R., Wang, S. X., et al. 2020, A\&A, 636, A58

Ballard, S. 2019, AJ, 157, 113

Barclay, T., Pepper, J., \& Quintana, E. V. 2018, ApJS, 239, 2

Batalha, N. E., Lewis, T., Fortney, J. J., et al. 2019, ApJL, 885, L25

Blake, C. H., Bloom, J. S., Latham, D. W., et al. 2008, PASP, 120, 860

Borucki, W. J., Koch, D., Basri, G., et al. 2010, Sci, 327, 977

Bowler, B. P., Liu, M. C., Shkolnik, E. L., \& Tamura, M. 2015, ApJS, 216, 7

Brown, T. M., Baliber, N., Bianco, F. B., et al. 2013, PASP, 125, 1031

Buckley, D. A. H., Swart, G. P., \& Meiring, J. G. 2006, Proc. SPIE, 6267, $62670 Z$

Burrows, A., Heng, K., \& Nampaisarn, T. 2011, ApJ, 736, 47

Chabrier, G., \& Baraffe, I. 2000, ARA\&A, 38, 337

Chen, J., \& Kipping, D. 2017, ApJ, 834, 17

Cloutier, R., Astudillo-Defru, N., Bonfils, X., et al. 2019, A\&A, 629, A111

Collins, K. A., Kielkopf, J. F., Stassun, K. G., \& Hessman, F. V. 2017, AJ, 153,77

Cowan, N. B., \& Agol, E. 2011, ApJ, 729, 54

Crause, L. A., Sharples, R. M., Bramall, D. G., et al. 2014, Proc. SPIE, 9147, $91476 \mathrm{~T}$

Crossfield, I. J. M., Waalkes, W., Newton, E. R., et al. 2019, ApJL, 833, L16

Foreman-Mackey, D. 2016, JOSS, 1, 24

Foreman-Mackey, D., Hogg, D. W., Lang, D., \& Goodman, J. 2013, PASP, 125,306

Gaia Collaboration, Brown, A. G. A., Vallenari, A., et al. 2018, A\&A, 616, A1

Gillon, M., Jehin, E., Fumel, A., Magain, P., \& Queloz, D. 2013, EPJWC, 47, 03001

Goldreich, P., \& Soter, S. 1966, Icar, 5, 375

Günther, M. N., Pozuelos, F. J., Dittmann, J. A., et al. 2019, NatAs, 3, 1099

Hogg, D. W., \& Foreman-Mackey, D. 2018, ApJS, 236, 11

Howell, S. B., Sobeck, C., Haas, M., et al. 2014, PASP, 126, 398

Hunter, J. D. 2007, CSE, 9, 90

Ingersoll, A. P. 2013, Planetary Climates (Princeton, NJ: Princeton Univ. Press)

Jeans, J. H. 1905, Natur, 71, 607

Jehin, E., Gillon, M., Queloz, D., et al. 2011, Msngr, 145, 2

Jenkins, J. M. 2002, ApJ, 575, 493

Jenkins, J. M., Twicken, J. D., McCauliff, S., et al. 2016, Proc. SPIE, 9913, 99133E

Jensen, E. 2013, Tapir: A Web Interface for Transit/Eclipse Observability, Astrophysics Source Code Library, ascl:1306.007

Kempton, E. M. R., Bean, J. L., Louie, D. R., et al. 2018, PASP, 130, 114401

Kniazev, A. Y., Gvaramadze, V. V., \& Berdnikov, L. N. 2016, MNRAS, 459, 3068

Kniazev, A. Y., Gvaramadze, V. V., \& Berdnikov, L. N. 2017, in Stars: From Collapse to Collapse, ed. Yu. Balega et al., Vol. 510 (San Fransisco, CA: ASP), 480

Kopparapu, R. K., Ramirez, R., Kasting, J. F., et al. 2013, ApJ, 765, 131

Kopparapu, R. k., Wolf, E. T., \& Meadows, V. S. 2019, arXiv:1911.04441

Kostov, V. B., Schlieder, J. E., Barclay, T., et al. 2019, AJ, 158, 32

Kotani, T., Tamura, M., Suto, H., et al. 2014, Proc. SPIE, 9147, 914714

Kreidberg, L. 2015, PASP, 127, 1161

Laughlin, G., Bodenheimer, P., \& Adams, F. C. 1997, ApJ, 482, 420

Li, J., Tenenbaum, P., Twicken, J. D., et al. 2019, PASP, 131, 024506

Lightkurve Collaboration, Cardoso, J. V. d. M., Hedges, C., et al. 2018, Lightkurve: Kepler and TESS time series analysis in Python, Astrophysics Source Code Library, ascl:1812.013

Luque, R., Pallé, E., Kossakowski, D., et al. 2019, A\&A, 628, A39

Mahadevan, S., Ramsey, L., Bender, C., et al. 2012, Proc. SPIE, 8446, 84461S

Mahadevan, S., Ramsey, L. W., Terrien, R., et al. 2014, Proc. SPIE, 9147, $91471 \mathrm{G}$

Mandel, K., \& Agol, E. 2002, ApJL, 580, L171

Mann, A. W., Dupuy, T., Kraus, A. L., et al. 2019, ApJ, 871, 63

Mann, A. W., Feiden, G. A., Gaidos, E., Boyajian, T., \& von Braun, K. 2015, ApJ, 804, 64 
McCully, C., Turner, M., Volgenau, N., et al. 2018, LCOGT/banzai: Initial Release, v0.9.4, Zenodo, doi:10.5281/zenodo. 1257560

McKinney, W. 2011, in Proc. 9th Python in Science Conf., ed. S. van der Walt \& J. Millman (Austin, TX: SciPy), 14

Mulders, G. D., Pascucci, I., \& Apai, D. 2015, ApJ, 814, 130

Newton, E. R., Irwin, J., Charbonneau, D., et al. 2016, ApJ, 821, 93

Newton, E. R., Irwin, J., Charbonneau, D., et al. 2017, ApJ, 834, 85

Nutzman, P., \& Charbonneau, D. 2008, PASP, 120, 317

Parviainen, H., \& Aigrain, S. 2015, MNRAS, 453, 3821

Parviainen, H., Tingley, B., Deeg, H. J., et al. 2019, A\&A, 630, A89

Prša, A., Harmanec, P., Torres, G., et al. 2016, AJ, 152, 41

Quirrenbach, A., Amado, P. J., Mandel, H., et al. 2010, Proc. SPIE, 7735, 773513

Rajpurohit, A. S., Reylé, C., Allard, F., et al. 2013, A\&A, 556, A15

Ricker, G. R., Winn, J. N., Vanderspek, R., et al. 2015, JATIS, 1, 014003

Rucinski, S. M. 1992, AJ, 104, 1968

Seager, S., \& Mallén-Ornelas, G. 2003, ApJ, 585, 1038

Shields, A. L., Bitz, C. M., \& Palubski, I. 2019, ApJL, 884, L2

Simcoe, R. A., Burgasser, A. J., Bernstein, R. A., et al. 2008, Proc. SPIE, 7014, $70140 \mathrm{U}$
Smith, J. C., Stumpe, M. C., Van Cleve, J. E., et al. 2012, PASP, 124, 1000 Sozzetti, A., Torres, G., Charbonneau, D., et al. 2007, ApJ, 664, 1190

Stassun, K. G., Oelkers, R. J., Paegert, M., et al. 2019, AJ, 158, 138

Stefansson, G., Cañas, C., Wisniewski, J., et al. 2020, AJ, 159, 100

Stumpe, M. C., Smith, J. C., Catanzarite, J. H., et al. 2014, PASP, 126, 100

Stumpe, M. C., Smith, J. C., Van Cleve, J. E., et al. 2012, PASP, 124, 985

Sullivan, P. W., Winn, J. N., Berta-Thompson, Z. K., et al. 2015, ApJ, 809, 77

Tofflemire, B. M., Mathieu, R. D., \& Johns-Krull, C. M. 2019, AJ, 158, 245

Tokovinin, A. 2018, PASP, 130, 035002

Twicken, J. D., Catanzarite, J. H., Clarke, B. D., et al. 2018, PASP, 130 , 064502

van der Walt, S., Colbert, S. C., \& Varoquaux, G. 2011, CSE, 13, 22

VanderPlas, J. T. 2018, ApJS, 236, 16

Vanderspek, R., Huang, C. X., Vanderburg, A., et al. 2019, ApJL, 871, L24

West, A. A., Hawley, S. L., Bochanski, J. J., et al. 2008, AJ, 135, 785

Winters, J. G., Medina, A. A., Irwin, J. M., et al. 2019, AJ, 158, 152

Zahnle, K. J., \& Catling, D. C. 2017, ApJ, 843, 122

Ziegler, C., Tokovinin, A., Briceño, C., et al. 2020, AJ, 159, 19 